\title{
Physician-Delivered Injection Therapies for Mechanical Neck Disorders: A Systematic Review Update (Non-Oral, Non-Intravenous Pharmacological Interventions for Neck Pain)
}

\author{
Anita R. Gross ${ }^{*}, 1$, Paul M. Peloso ${ }^{2}$, Erin Galway ${ }^{1}$, Neenah Navasero ${ }^{1}$, Karis Van Essen ${ }^{1}$, \\ Nadine Graham ${ }^{1}$, Charlie H Goldsmith ${ }^{3}$, Wisam Gzeer ${ }^{1}$, Qiyun Shi $^{4}$, Ted Haines ${ }^{1}$ and COG $^{\S}$ \\ ${ }^{1}$ McMaster University, Hamilton, Canada \\ ${ }^{2}$ Merck, Kenilworth, NJ, USA \\ ${ }^{3}$ Simon Fraser University, Burnaby, Canada \\ ${ }^{4}$ Western University, London, Canada
}

Abstract: Background: Controversy persists regarding medicinal injections for mechanical neck disorders (MNDs).

Objectives: To determine the effectiveness of physician-delivered injections on pain, function/disability, quality of life, global perceived effect and patient satisfaction for adults with MNDs.

Search Methods: We updated our previous searches of CENTRAL, MEDLINE and EMBASE from December 2006 through to March 2012.

Selection Criteria: We included randomized controlled trials of adults with neck disorders treated by physician-delivered injection therapies.

Data Collection and Analysis: Two authors independently selected articles, abstracted data and assessed methodological quality. When clinical heterogeneity was absent, we combined studies using random-effects models.

Results: We included 12 trials (667 participants). No high or moderate quality studies were found with evidence of benefit over control. Moderate quality evidence suggests little or no difference in pain or function/disability between nerve block injection of steroid and bupivacaine $v s$ bupivacaine alone at short, intermediate and long-term for chronic neck pain. We found limited very low quality evidence of an effect on pain with intramuscular lidocaine $v s$ control for chronic myofascial neck pain. Two low quality studies showed an effect on pain with anaesthetic nerve block $v s$ saline immediately post treatment and in the short-term. All other studies were of low or very low quality with no evidence of benefit over controls.

Authors' Conclusions: Current evidence does not confirm the effectiveness of IM-lidocaine injection for chronic mechanical neck pain nor anaesthetic nerve block for cervicogenic headache. There is moderate evidence of no benefit for steroid blocks $v s$ controls for mechanical neck pain.

Keywords: Injections, neck, pain, systematic review, meta-analysis.

\section{INTRODUCTION}

Neck disorders are common and can be disabling and costly $[1,2]$. The prevalence of neck pain in the general population varies from 146 to 213 per 1000 people [2]. The prevalence of neck pain is higher in women and increases with age with a peak between 30 to 45 years $[1,3-5]$. Subjects reporting neck pain in the previous year also reported recurrence in $93.7 \%$ [5]. Each year $0.6 \%$ of adults

*Address correspondence to this author at the McMaster University, School of Rehabilitation Science, IAHS, 1400 Main Street West, 4th Floor, Hamilton, ON, L8S 1C7, Canada; Tel: 905-577-0098; Fax: 905-577-0603;

E-mail: grossa@mcmaster.ca

${ }^{\S} \mathrm{COG}$, Cervical Overview Group is an international research group that conducts and maintains systematic reviews for neck pain. living in Saskatchewan, Canada reported developing disabling neck pain and $37.3 \%$ of patients report persistent pain [6]. Twenty-four to $50 \%$ of people have persistent symptoms 12 months after a motor vehicle accident [7, 8]. It appears that women and those with a depressed mood have a higher risk of developing persistent widespread pain following whiplash associated disorder (WAD) [9]. Neck pain accounts for approximately $11.3 \%$ of all Workplace Safety and Insurance Board lost time claims in Ontario [1]. In the United States total health care costs for neck pain were estimated to be $\$ 686$ million in 1996 dollars [10]. Physiciandelivered injections are often used to treat mechanical neck pain presenting with or without radicular symptoms. These can include several routes, including subcutaneous, intramuscular, intra-articiular, intera-thecal, etc. However evidence for the safest and most effective injections is lacking [11]. 


\section{Description of the Condition}

In this review we considered patients with mechanical neck disorders (MNDs) including neck related diagnoses of: sprains and strains, WAD, neck pain associated with work related injury, neck pain associated with myofascial pain, neck pain with associated degenerative changes, and headaches of cervicogenic origin. Mechanical neck disorders can be classified as specific or non-specific neck pain. Specific neck pain results from an identifiable pain generating mechanism such as the intervertebral disc, cervical facet joints, and nerve root dura. It may present with radicular pain, somatic pain including discogenic pain and facet joint pain. Non-specific neck pain has no identifiable aetiology although it should be aggravated by neck movements. There are many possible sources of nonspecific neck pain including but not limited to intervertebral disc, cervical facet joints, atlanto-axial and atlanto-occipital joints, ligaments, fascia, muscles and nerve root dura. We classified the mechanical neck pain duration as acute if it was present for less than 30 days, subacute if it was present between 30 and 90 days or chronic if it was present for longer than 90 days.

\section{Description of the Intervention and how it Might Work}

Corticosteroids are thought to act to reduce inflammation by inhibiting phospholipase A and thereby the downstream synthesis of pro-inflammatory molecules. However a 2012 study suggests steroids may have more of an effect in reducing pain than inflammation [12].

Local anaesthetic agents produce a reversible loss of sensation in the injected muscle or joint by interrupting the conduction of impulses in peripheral nerves. In general, all effects are reversible with amide anaesthetic agents having a longer duration of effect as compared to ester anesthetic agents [13].

Some agents, such as dry needling and saline injections which are chemically neutral, are thought to exert their effects through mechanical means, by disruption of a local muscular spasm and not through specific local biochemical alternations. Subcutaneous agents such as $\mathrm{CO}_{2}$ may work through local mechanical disruption, although some have suggested that the acidosis induced may alter local blood flow. For some injected therapies, the exact mechanism of action has not been well-established. These injected agents are typically applied to the neck structures that are suspected of causing the pain, or to the referred areas of pain associated with the neck pain, such as myofascial trigger points. These injections are generally expected to exert their effects on the tissues locally.

Other agents that might be delivered via intramuscular or intravenous injection to treat neck pain, including opioids (e.g. morphine), non-steroidal anti-inflammatory medications (e.g. toradol), muscle relaxants (e.g. diazepam), etc. were excluded, since these agents are thought to work through achieving therapeutic systemic levels of the drug or central effects and these agents are not typically applied to the neck tissues directly with the intent of inducing local effects.

\section{Why it is Important to do this Review}

In our last review on medicinal and injection therapies Peloso 2007 (search end date 2006) [14], we found evidence from one trial that intramuscular injection of lidocaine (IMlidocaine) was superior to placebo for chronic neck disorders at short-term follow-up. Another trial showed that IM-lidocaine was similar to ultrasound for chronic neck pain at short-term follow-up.

A 2011 systematic review on botolinum toxin (BoNT) for subacute/chronic neck pain was updated [15] (search end date 2010) and the conclusions were consistent with an International Collaboration on Neck (ICON) overview of systematic reviews on medicines and injections for neck pain [16] in suggesting that there is evidence against the use of BoNT-A for chronic neck pain or subacute/chronic whiplash. Further the ICON review of systematic reviews gave evidence against medial branch block with steroids for chronic facet joint pain, but for the use of eperison hydrochloride for chronic neck pain. In the Peloso 2012 [16] review of reviews it was noted that the search update for lidocaine and epidural ended in 2008 while the searches for steroids, nerve blocks and insufflation ended in 2006. During this 6 year gap additional trials have been published on medicinal injections for neck pain; therefore, an update literature search was warranted.

\section{OBJECTIVES}

Our systematic review assessed the treatment effectiveness of medical injections in acute, subacute and chronic MNDs. Outcomes of interest included pain, function/disability, quality of life, global perceived effect and patient satisfaction in different timeframes.

\section{METHODS}

Methods consistent with the series of Cervical Overview Group (COG) reviews were utilized here as well. This group publishes both Cochrane and other Systematic Reviews. As such the searches meet the standards recommended by the Cochrane Collaboration Back Group.

\section{CRITERIA FOR CONSIDERING STUDIES FOR THIS REVIEW}

\section{Types of Studies}

We included all published randomized controlled trials (RCTs). We had no restrictions based on the methodological quality of RCTs.

\section{Types of Participants}

We included trials of adults ( $\geq 18$ years) with a spectrum of symptom duration (acute $<30$ days, subacute 30 to 90 days, or chronic $>90$ days) who had:

1. Neck pain without radicular findings, including nonspecific (mechanical, simple) neck pain of unknown etiology or whiplash associated disorder (WAD) grade I to II.

2. Neck pain with associated myofascial pain syndrome and neck pain with associated degenerative changes.

3. Neck pain with cervicogenic headache.

4. Neck pain with radicular findings suggesting a nerve root impingement.

5. Neck pain with radicular findings, including degenerative joint or disc disease with spinal stenosis, spondylolisthesis, discogenic radiculopathy, or WAD grade III. 
We excluded studies of neck disorders with the following specific causes:

1. Definite or possible long tract signs (e.g. myelopathies).

2. Neck pain caused by other pathological entities.

3. Headache not of cervical origin but associated with the neck pain.

4. Co-existing headache when either neck pain was not dominant or the headache was not provoked by neck movements or sustained neck postures.

5. 'Mixed' headache, which includes more than one headache classification.

6. WAD grade IV.

The above inclusion and exclusion criteria are based on criteria used by the Cervical Overview Group (COG) in the systematic review on BoNT for subacute/chronic neck pain update [15].

\section{Types of Interventions}

We included studies using physician-delivered injections compared to placebo or control. A physician-delivered injection was defined as one administered by a physician to a muscle, joint or any part of the body through a syringe or a medical device. Although typically applied to the cervical region, the injections could also be applied to other body regions as long as the injection was given with the intent of treating the local effects of neck pain. For example patients with neck pain may have referred pain with myofascial trigger points, and these trigger points are often injected as well. Typical medications for injection were local anaesthetics, corticosteroids, and BoNT. However as we have recently updated the review on BoNT [15], it is not considered further in this review. Further, we did not consider therapies delivered by injection that are expected to work by achieving systemic levels of drug (like opioids, nonsteroidals, muscle relaxants) since these agents are not typically delivered into the neck tissues or myofascial trigger points.

Interventions were contrasted against the following comparisons:

- $\quad$ Physician-delivered injection $v s$ sham or placebo (e.g. intra-muscular lidocaine $v s$ dry needling);

- Physician-delivered injection $v s$ waitlist or no treatment;

- Physician-delivered injection plus another intervention $v s$ that same intervention (e.g. intramuscular lidocaine + stretching $v s$ stretching);

\section{Types of Outcome Measures}

We were interested in outcomes of pain, function/disability, quality of life, global perceived effect and patient satisfaction. Function and disability could be measured using either self-report measures or observer-based physical performance tests $[17,18]$. Some examples of pain outcome measures are: the Visual Analogue Scale (VAS) and the Numeric Pain Rating Scale (NPRS). A representative example of a disability and function outcome measure is the patient completed Neck Disability Index (NDI). Due to their limited clinical agreement (and hence their uncertain utility as outcome measures), tests used during a standard physical examination, such as inspection, range of motion, strength, palpation, provocation, muscular stability, neurological tests, and cervical proprioception were excluded from this review. Data on adverse effects and cost of treatments were captured when they were reported. The duration of follow-up was defined as: immediately post treatment (within one day); short-term follow-up (closest to four weeks); intermediateterm follow-up (closest to six months); and long-term follow-up (closest to 12 months).

\section{SEARCH METHODS FOR IDENTIFICATION OF STUDIES}

\section{Electronic Searches}

A research librarian searched bibliographic databases, without language restrictions, in the medical, chiropractic, and allied health literature, through Cochrane Central Register of Controlled Trials (CENTRAL; The Cochrane Library 2012, issue 1) and MEDLINE, EMBASE from 2006 to March 2012; data from our previous Cochrane review [15] with a search from root to 2006 were also included in this review. See APPENDIX A for the search strategy for MEDLINE.

\section{Searching Other Resources}

We screened references and examined the review team's personal files as well as key conference proceedings to identify other potential references.

\section{DATA COLLECTION AND ANALYSIS}

\section{Selection of Studies}

At least two authors with expertise in medicine and physiotherapy independently identified citations, selected studies, and reached consensus. Agreement for study selection was assessed using the quadratic weighted Kappa statistic (Kw) [19]. A third author was consulted in case of persisting disagreement.

\section{Data Extraction and Management}

At least two authors independently conducted data extraction. Pre-piloted forms were used for all phases, except for the evaluation of clinical applicability, where we followed the recommendations of Furlan 2009 [20].

\section{Assessment of Risk of Bias in Included Studies}

The COG used a calibrated team of assessors. At least two assessors independently assessed the risk of bias (Table 1). Risk of bias tables were presented and discussed by the broader COG validity assessment team to maximize inter-rater reliability [21]. Consensus was reached on the final presented risk of bias assessments following the updated Cochrane criteria [22]. Studies that met 6 or more of a maximum 12 criteria were considered to have low risk of bias and studies that met less than 6 were considered to have high risk of bias. The following characteristics for risk of bias were assessed: randomization, concealment of treatment allocation, withdrawal/drop-out rate, intention-to-treat analysis, selective outcome reporting, similar at baseline, similar co-interventions, acceptable compliance, similar timing of assessment, and blinding of patient, provider, and outcome assessor. Explicit details on study design were extracted including: number of patients analyzed and 
randomized, use of intention-to-treat analysis, and power analysis.

\section{Measures of Treatment Effect}

We used Revman version 5.0.14 to conduct statistical analysis. Descriptive statistics provided a summarized description of the patient groups, interventions, outcomes, adverse effect of treatments, and cost of care. All results reported were based on the sample size analysed using the 'intention-to-treat' principle. The Cochrane Back Review Group guidelines [20] were used to estimate minimum clinically important differences (MCIDs). For pain, it was assumed that the MCID was 10 points on a 100-point pain intensity scale [2325]. For the neck disability index, a MCID of 7 of 50 neck disability index units were used [25, 26]. For other outcomes (i.e. global perceived effect and quality of life scales) where there was an absence of clear guidance on the size of a clinically important effect sizes, we used the common hierarchy of Cohen 1988 that discussed effect sizes as: small (0.20), medium (0.50) or large (0.80). For continuous outcomes reported as medians, we calculated effect sizes based on Kendal 1963 [27].

\section{Unit of Analysis Issues}

For continuous data, standardized mean differences with 95\% confidence intervals (SMD; 95\% CI) were used, since different measures were frequently used to address the same clinical outcome. We calculated relative risks (RR) for dichotomous outcomes. A relative risk less than one represents treatment benefit. When neither continuous nor dichotomous data were available, we extracted the study results and statistical significance as reported by the author(s) in the original study report and we noted these results in the characteristics of included studies table (Table 2).

Table 1. Risk of Bias

\begin{tabular}{|c|c|c|c|c|c|c|c|c|c|c|c|c|c|}
\hline Study & $\begin{array}{l}\text { Random } \\
\text { Adequate } \\
\text { (A) }\end{array}$ & $\begin{array}{l}\text { Allocation } \\
\text { Concealed } \\
\text { (B) }\end{array}$ & $\begin{array}{l}\text { Patient } \\
\text { Blind } \\
\text { (C) }\end{array}$ & $\begin{array}{l}\text { Care } \\
\text { Provider } \\
\text { Blind } \\
\text { (D) }\end{array}$ & $\begin{array}{c}\text { Assessor } \\
\text { Blind } \\
\text { (E) }\end{array}$ & $\begin{array}{l}\text { Drop Outs } \\
\text { (F) }\end{array}$ & $\begin{array}{c}\text { All } \\
\text { Analyzed } \\
\text { (G) }\end{array}$ & $\begin{array}{c}\text { Selective } \\
\text { Outcome } \\
\text { (H) }\end{array}$ & $\begin{array}{c}\text { Baseline } \\
\text { Similar } \\
\text { (I) }\end{array}$ & $\begin{array}{c}\text { Co- } \\
\text { Intervent. } \\
\text { Avoided } \\
\text { (J) }\end{array}$ & $\begin{array}{c}\text { Compliance } \\
\text { Acceptable } \\
\text { (K) }\end{array}$ & $\begin{array}{c}\text { Timing } \\
\text { of } \\
\text { Outcome } \\
\text { (L) }\end{array}$ & Total \\
\hline $\begin{array}{l}\text { Anderberg } \\
2007 \text { [41] }\end{array}$ & $?$ & $?$ & + & 0 & + & + & + & $?$ & 0 & $?$ & + & + & 6 \\
\hline $\begin{array}{c}\text { Ay } 2010 \\
{[31]}\end{array}$ & $?$ & $?$ & $?$ & $?$ & $?$ & + & + & $?$ & + & $?$ & $?$ & + & 4 \\
\hline $\begin{array}{l}\text { Brockow } \\
2008 \text { [39] }\end{array}$ & + & + & 0 & 0 & 0 & + & + & $?$ & 0 & $?$ & $?$ & + & 5 \\
\hline $\begin{array}{l}\text { Esenyel } \\
2000 \text { [33] }\end{array}$ & $?$ & $?$ & 0 & 0 & 0 & $?$ & $?$ & $?$ & $?$ & $?$ & $?$ & + & 1 \\
\hline $\begin{array}{c}\text { Ferrante } \\
1998 \text { [37] }\end{array}$ & $?$ & $?$ & + & + & + & $?$ & $?$ & $?$ & + & $?$ & $?$ & + & 5 \\
\hline $\begin{array}{c}\text { Hong } \\
1994 \text { [34] }\end{array}$ & $?$ & $?$ & 0 & 0 & 0 & $?$ & $?$ & $?$ & 0 & $?$ & $?$ & 0 & 0 \\
\hline $\begin{array}{c}\text { Kamanli } \\
2005 \text { [32] }\end{array}$ & $?$ & $?$ & $?$ & 0 & $?$ & $?$ & $?$ & $?$ & 0 & $?$ & $?$ & + & 1 \\
\hline $\begin{array}{l}\text { Manchikanti } \\
\text { 2010a [28-30] }\end{array}$ & + & $?$ & + & + & + & + & + & $?$ & + & $?$ & $?$ & + & 8 \\
\hline $\begin{array}{c}\text { Manchikanti } \\
\text { 2010b [38] }\end{array}$ & + & ? & + & + & + & $?$ & 0 & $?$ & $?$ & $?$ & $?$ & + & 5 \\
\hline $\begin{array}{c}\text { Naja } \\
2006[35]\end{array}$ & + & + & + & + & + & 0 & 0 & $?$ & + & $?$ & + & + & 8 \\
\hline $\begin{array}{c}\text { Sand } \\
1992[40]\end{array}$ & $?$ & $?$ & $?$ & $?$ & $?$ & $?$ & $?$ & $?$ & $?$ & $?$ & + & + & 2 \\
\hline $\begin{array}{c}\text { Terzi } \\
2002[36]\end{array}$ & $?$ & $?$ & + & + & + & + & + & $?$ & + & + & + & + & 9 \\
\hline
\end{tabular}

Key:

$+=$ Yes, item adequately addressed.

$0=$ No, not adequately addressed.

$?=$ Unsure if adequately addressed.

A Was the method of randomization adequate?

B Was the treatment allocation concealed?

C Was the patient blinded to the intervention?

D Was the care provider blinded to the intervention?

E Was the outcome assessor blinded to the intervention?

F Was the dropout rate described and acceptable?

$\mathrm{G}$ Were all randomised participants analysed in the group to which they were allocated?

$\mathrm{H}$ Are the reports of the study free of suggestion of selective outcome reporting?

I Were the groups similar at baseline regarding the most important prognostic indicators?

J Were co-interventions avoided or similar?

$\mathrm{K}$ Was the compliance acceptable in all groups?

L Was the timing of the outcome assessment similar in all groups? 
Table 2. Characteristics of Included Studies

\begin{tabular}{|c|c|c|}
\hline Author/Method/Participants & Intervention & Outcomes/Notes \\
\hline $\begin{array}{l}\text { Ay } 2010 \text { [31] } \\
\text { Method: } \\
\text { RCT } \\
\text { N(A/R): } 40 / 40 \\
\text { Power Analysis: Done (power } 0.95 \text { ) } \\
\text { Intention-to-treat Analysis: NA } \\
\text { Participants: } \\
\text { Subacute trigger point myofascial pain syndrome }\end{array}$ & $\begin{array}{l}\text { INDEX TREATMENT } \\
\text { Lidocaine injection }(\mathrm{LiC}) \text { : } 1 \% \mathrm{LiC} \text { injection; timing } \\
\text { and frequency = single injection at the start of the } \\
\text { study, dose }=2 \mathrm{ml} \text {, route = intramuscular to trapezius } \\
\text { muscle } \\
\text { COMPARISON TREATMENT } \\
\text { Dry Needling (DNG); timing and frequency = single } \\
\text { injection at the start of the study, route = intramuscular } \\
\text { to trapezius muscle } \\
\text { COINTERVENTION: home based exercise program } \\
\text { (isometric-isotonic neck exercises and back extensor } \\
\text { stretching exercises every day for } 12 \text { weeks) } \\
\text { Duration of treatment: } 1 \text { session } \\
\text { Duration of follow-up: } 12 \text { weeks }\end{array}$ & $\begin{array}{l}\text { PAIN: VAS (0: no pain - 10: worst pain) } \\
\text { Baseline: LiC: } 5.82 \text {, DNG: } 5.55 \\
\text { End of Study Mean: LiC: } 0.97, \text { DNG: } 1.25 \\
\text { Report Results: no significant difference between } \\
\text { groups } \\
\text { SMD -2.00 (95\% CI Random: 0.54,-1.46) } \\
\text { DEPRESSION: BDI (lower indicates less } \\
\text { depression, max score 63) } \\
\text { Baseline: LiC: } 14.52, \text { DNG: } 12.12 \\
\text { End of Study Mean: LiC: } 9.92 \text {, DNG: } 10.10 \\
\text { Report Results: no significant difference between } \\
\text { groups } \\
\text { SIDE EFFECTS: NR } \\
\text { COST OF CARE: NR }\end{array}$ \\
\hline $\begin{array}{l}\text { Brockow 2008 [39] } \\
\text { Method: } \\
\text { RCT } \\
\text { N(A/R): } 63 / 63 \\
\text { Power Analysis: done } \\
\text { Intention-to-treat: done } \\
\text { Participants: } \\
\text { Acute nonspecific neck pain }\end{array}$ & $\begin{array}{l}\text { INDEX TREATMENT } \\
\text { Subcutaneous Carbon Dioxide Insufflation (SCDI): } \\
\text { Timing and frequency = 3x/week to a maximun of } 9 \\
\text { treatments at the site of muscle tenderness; dose = } \\
25 \mathrm{ml} \mathrm{CO}_{2} \text { was administered per tender site to a } \\
\text { maximum of } 100 \mathrm{ml} \text {, If }>4 \text { tender sites, the sites of } \\
\text { maximum tenderness were insufflated; route = } \\
\text { subcutaneous insufflation to } 4 \text { cervical tenderness sites } \\
\text { COMPARISON TREATMENT } \\
\text { Sham Ultrasound (US); Timing and frequency = } \\
3 \text { x/week to a maximum of } 9 \text { treatments at the site of } \\
\text { muscle tenderness; dose }=1.5 \mathrm{~cm}^{2} \text { transducer for } 5 \\
\text { minutes with intensity of } 0.2 \text { Watt per cm }{ }^{2} \text { displayed } \\
\text { on the sham device; route }=\text { stationary with no pressure } \\
\text { at the } 4 \text { sites of cervical muscle tenderness } \\
\text { CO-INTERVENTION: local infrared light } 100 \\
\text { Watts at } 25-30 \mathrm{~cm} \text { at same number and frequency } \\
\text { as Subcutaneous Insufflation or sham Ultrasound } \\
\text { for } 10 \text { min/session, }<175 \mathrm{mg} \text { diclofenac each } \\
\text { morning } \\
\text { Duration of treatment: maximum } 9 \text { treatments } \\
\text { (maximum } 3 \text { weeks). } \\
\text { Duration of follow-up: } 7 \text { days }\end{array}$ & $\begin{array}{l}\text { PAIN INTENSITY (VAS } 0 \text { to } 100 \mathrm{~mm} \text { ) } \\
\text { Baseline: SCDI: 68.7, Sham US: } 69.4 \\
\text { End of Study Mean: SCDI: 33, Sham US: } 30.9 \\
\text { Reported Results: no significant difference } \\
\text { between groups } \\
\text { SMD } 0.07 \text { (95\% CI Random: }-0.28 \text { to } 0.42 \text { ) } \\
\text { AFFECTIVE PAIN [German MPQ) scores of } 14 \\
\text { to 56, lower score = better] } \\
\text { Baseline: SCDI: 32.7, Sham US: } 34.7 \\
\text { End of Study Mean: SCDI: 20.2, Sham US: } 21.9 \\
\text { Reported Results: no significant difference } \\
\text { between groups } \\
\text { SENSORY PAIN (MPQ, score of } 10 \text { to } 14 \text {, lower } \\
\text { score = better) } \\
\text { Baseline: SCDI: 18.2, Sham US: } 13.4 \\
\text { End of Study Mean: SCDI: } 19.5 \text {, Sham US: } 13.8 \\
\text { Reported Results: no significant difference between } \\
\text { groups } \\
\text { SIDE EFFECTS: no adverse events reported } \\
\text { COST OF CARE: NR }\end{array}$ \\
\hline $\begin{array}{l}\text { Esenyel 2000 [33] } \\
\text { Method: } \\
\text { RCT } \\
\text { N(A/R): 90/108 } \\
\text { Power Analysis: NR } \\
\text { Intention-to-treat Analysis: NA } \\
\text { Participants: } \\
\text { Chronic mechanical neck pain (myofascial pain) }\end{array}$ & $\begin{array}{l}\text { INDEX TREATMENT } \\
\text { Lidocaine (LiC): timing = NR; frequency = } 1 \\
\text { injection; dose = } 1 \% \text { lidocaine; duration = } 1 \text { session; } \\
\text { route = intramuscular injection of upper trapezius; plus } \\
\text { neck stretching exercises } \\
\text { COMPARISON TREATMENT } \\
\text { Ultrasound (US): dose = } 1.5 \text { w/cm squared; } 10 \\
\text { sessions of } 6 \text { minutes each; plus neck stretching } \\
\text { exercises } \\
\text { CONTROL (Cntl): neck stretching exercise } \\
\text { CO-INTERVENTION: NR } \\
\text { Duration of treatment: LiC } 1 \text { session; US } 10 \text { sessions; } \\
\text { Cntl } 10 \text { sessions } \\
\text { Duration of follow-up: } 3 \text { months }\end{array}$ & $\begin{array}{l}\text { PAIN INTENSITY (VAS 0 to 10) } \\
\text { Baseline Mean: LiC 7.16, US 7.24, Cntl 6.50 } \\
\text { End of Study Mean: LiC 3.19, US 3.08, Cntl } 5.76 \\
\text { Absolute Benefit: LiC 3.97, US 4.16, Cntl } 0.72 \\
\text { Reported Results: significant, favouring Lidocaine } \\
\text { over Cntl; not significant for LiC vs US } \\
\text { SMD(LiC v Cntl): }-1.36 \text { (95\% CI Random: }-1.93 \text { to } \\
-0.80 \text { ) } \\
\text { SMD(LiC v US): 0.04(95\% CI Random: }-0.46 \text { to } \\
\text { 0.55) (power 8\%) } \\
\text { SIDE EFFECTS: NR } \\
\text { COST OF CARE: NR }\end{array}$ \\
\hline
\end{tabular}




\begin{tabular}{|c|c|c|}
\hline Author/Method/Participants & Intervention & Outcomes/Notes \\
\hline $\begin{array}{l}\text { Ferrante } 1998 \text { [37] } \\
\text { Method: } \\
\text { RCT (cross-over trial) } \\
\text { N(A/R): } 23 / 23 \\
\text { Power Analysis: NR } \\
\text { Intention-to-treat Analysis: NA } \\
\text { Participants: } \\
\text { Chronic mechanical neck disorder (myofascial } \\
\text { pain) }\end{array}$ & $\begin{array}{l}\text { INDEX TREATMENT } \\
\text { Sphenopalatine ganglion block (SPGB): timing and } \\
\text { dose = week } 1 \text { SPGB with } 4 \% \text { lidocaine, then week } \\
2 \text { trigger point injection with } 3 \mathrm{ml} 1 \% \text { lidocaine, } \\
\text { finally week } 3 \text { SPGB with saline placebo, } \\
\text { frequency = } 1 \text { session, duration = } 20 \text { minutes, route } \\
=\text { injection to trigger points of specific neck } \\
\text { muscles } \\
\text { COMPARISON TREATMENT } \\
\text { Placebo: SPGB with saline } \\
\text { CO-INTERVENTION: pre-existent medication } \\
\text { were not stopped (antidepressants, nonsteroidal } \\
\text { anti-inflammatory drugs, etc.); patients were } \\
\text { permitted to engage in stretch and spray exercises, } \\
\text { and in prescribed physical therapy } \\
\text { Duration of treatment: } 1 \text { session } \\
\text { Duration follow-up: } 1 \text { week }\end{array}$ & $\begin{array}{l}\text { PAIN INTENSITY (VAS } 0 \text { to 100) } \\
\text { Baseline Mean: SPGB } 62.7 \text {, Placebo } 47.4 \\
\text { End of week 1 Mean: graphed unable to extract } \\
\text { Reported Results: SPGB not significantly } \\
\text { different from placebo and less effective than } \\
\text { trigger point injection } \\
\text { SIDE EFFECTS: NR } \\
\text { COST OF CARE: NR } \\
\text { NOTE: author was contacted and responded. - he } \\
\text { no longer has original data available. }\end{array}$ \\
\hline $\begin{array}{l}\text { Hong } 1994[34] \\
\text { Method: } \\
\text { RCT } \\
\text { N(A/R): } 41 / 58 \\
\text { Power Analysis: NR } \\
\text { Intention-to-treat Analysis: NA } \\
\text { Participants: } \\
\text { Chronic mechanical neck disorder with or } \\
\text { without headache and } \\
\text { radicular symptoms (myofascial pain) }\end{array}$ & $\begin{array}{l}\text { INDEX TREATMENT } \\
\text { Lidocaine injection }(\mathrm{LiC}) \text { : timing = midday; } \\
\text { frequency }=10 \text { to } 20 \text { injections at once; dose }= \\
0.05 \% \text { (total } 0.05 \times 20=1.0 \mathrm{ml} \text { ); duration }=1 \text { day; } \\
\text { route }=\text { intramuscular injection to myofascial } \\
\text { trigger points with at least one located in the upper } \\
\text { trapezius muscle } \\
\text { COMPARISON TREATMENT } \\
\text { Dry needling (DNG): timing = midday; frequency } \\
=10 \text { to } 20 \text { injections at once; dose }=0 \text { ml (dry); } \\
\text { duration = } 1 \text { day; route }=\text { intramuscular injection to } \\
\text { myofascial trigger points with at least one located } \\
\text { in the upper trapezius muscle } \\
\text { CO-INTERVENTION: NR } \\
\text { Duration of treatment: } 1 \text { day } \\
\text { Duration of follow-up: } 2 \text { weeks }\end{array}$ & $\begin{array}{l}\text { PAIN INTENSITY (0 to 10) } \\
\text { Baseline Mean: LiC 7.88, DNG 7.80 } \\
\text { End of Study Mean: LiC 0.96, DNG } 4.93 \\
\text { Absolute Benefit: LiC 6.92, DNG } 2.87 \\
\text { Reported Results: significant, favouring } \\
\text { Lidocaine } \\
\text { SMD at } 2 \mathrm{w} \text { follow-up: }-3.46 \text { (95\% CI Random: - } \\
4.46 \text { to -2.46) } \\
\text { SIDE EFFECTS: increased pain at injection site } \\
\text { COST OF CARE: NR }\end{array}$ \\
\hline $\begin{array}{l}\text { Kamanli 2005 [32] } \\
\text { Method: } \\
\text { RCT } \\
\text { N(A/R): 29/29 } \\
\text { Power Analysis: NR } \\
\text { Intention-to-treat Analysis: NA } \\
\text { Participants: } \\
\text { Chronic mechanical neck disorder (myofascial } \\
\text { pain), no radicular finding }\end{array}$ & $\begin{array}{l}\text { INDEX TREATMENTS } \\
\text { Lidocaine injection (LiC): timing \& frequency = } \\
\text { single injection; dose = } 1 \text { ml } 0.5 \% \text { lidocaine to each } \\
\text { trigger point; duration = minutes; route = } \\
\text { intramuscular technique modified from Travel \& } \\
\text { Simons; } 25 \text { gauge needle, } 1.25 \text { inch length; trigger } \\
\text { points were located on cervical, back, or shoulder } \\
\text { muscles (upper, lower, and middle trapezius, } \\
\text { levator scapula, teres minor, supraspinatus, } \\
\text { infraspinatus), } \\
\text { COMPARISON TREATMENT } \\
\text { Dry Needling (DNG): modification of technique } \\
\text { described by Travel \& Simons; frequency = } 1 \\
\text { session } \\
\text { Botulinum toxin type A (BoNT-A): timing \& } \\
\text { frequency = single injection; dose = } 10 \text { to } 20 \text { IU in } \\
1 \text { ml to each trigger point; duration = minutes; } \\
\text { route = intramuscular } \\
\text { CO-INTERVENTION: passive stretch, home } \\
\text { exercise and information on prevention of postural } \\
\text { problems given to all groups }\end{array}$ & $\begin{array}{l}\text { PAIN (VAS } 0 \text { to 10) } \\
\text { Baseline Mean: BoNT-A 6.1(1.70), LiC } \\
6.9(1.77) \text {, DNG 7.0(1.77) } \\
\text { Absolute Benefit: BoNT-A 3.4, LiC 5.0, DNG } \\
1.9 \\
\text { Reported Results: corrected value not significant } \\
\text { for all comparisons } \\
\text { SMD (BoNT-A v LiC): } 0.49 \text { (95\%CI:-0.42 to } \\
\text { 1.41) } \\
\text { SMD (BTX v DNG): - } 1.03(95 \% \text { CI:-2.01 to - } \\
\text { 0.06) SMD (LiG v DNG):-1.27(95\%CI:-2.25 to - } \\
\text { 0.29) } \\
\text { SMD (LiC v BTX):- 0.49(95\%CI:-1.41 to 0.42) } \\
\text { Work DISABILITY (VAS 0 to 10) } \\
\text { Baseline Mean: BTX 5.5, LiC 5.1, DNG 6.8 } \\
\text { Absolute Benefit: BTX 3.0, LiC 3.1, DNG 1.7 } \\
\text { Reported Results: corrected value not significant } \\
\text { for all comparisons } \\
\text { SMD (LiC v DNG): }-1.05(95 \% \text { CI: -2.00 to -0.10) } \\
\text { SMD (LiC v BTX): -0.21(95\%CI:-1.12 to 0.69) } \\
\text { (power 59\%) }\end{array}$ \\
\hline
\end{tabular}


(Table 2) contd......

\begin{tabular}{|c|c|c|}
\hline Author/Method/Participants & Intervention & Outcomes/Notes \\
\hline & $\begin{array}{l}\text { Duration of treatment: } 1 \text { session. Duration of follow-up: } 4 \\
\text { weeks }\end{array}$ & $\begin{array}{l}\text { QUALITY of LIFE (Nottingham Health Profile } 0 \text { to } \\
\text { 38) } \\
\text { Baseline Mean: BTX 16.6, LiC 18.5, DNG } 16.2 \\
\text { Absolute Benefit: BTX 6.4, LiC 12.1, DNG } 2.0 \\
\text { Reported Results: corrected value not significant for } \\
\text { all comparisons } \\
\text { SMD (LiC v DNG):-1.24(95\%CI:-2.22 to -0.27) } \\
\text { SMD (LiC v BTX):-0.71(95\%CI:-1.65 to 0.22) } \\
\text { (power 67\%) } \\
\text { Cervical ROM } \\
\text { Reported Results: No between study comparisons } \\
\text { reported } \\
\text { SIDE EFFECTS: Lidocaine = paraesthesia, burning; } \\
\text { BoNT-A = muscle pain, fatigue, headache } \\
\text { COST OF CARE: NR } \\
\text { NOTE : Baseline standard deviations were estimated } \\
\text { based on those providing (Lew, Ojala, Wheeler } \\
\text { (2001), Braker, Padberg) }\end{array}$ \\
\hline $\begin{array}{l}\text { Manchikanti 2010a [28-30] } \\
\text { Method: } \\
\text { RCT } \\
\text { N(A/R): 120/120 } \\
\text { Power Analysis: not done } \\
\text { Intention-to-treat Analysis: done } \\
\text { Participants: } \\
\text { Chronic non-specific neck pain }\end{array}$ & $\begin{array}{l}\text { INDEX TREATMENT } \\
\text { Cervical medial branch block with bupivacaine and } \\
\text { steroid (steroid); timing and frequency = first injection at } \\
\text { the start of the study, repeated based on response of } \\
\text { individual; } 0.5 \text { to } 1.0 \mathrm{~mL} \text { of mixture; route = cervical } \\
\text { medial branch block } \\
\text { COMPARISON TREATMENT } \\
\text { Cervical medial branch block with bupivacaine (non- } \\
\text { steroid); timing and frequency = first injection at the start } \\
\text { of the study, repeated based on response of individual; } \\
0.5 \text { to1.0 mL of mixture; route = cervical medial branch } \\
\text { block } \\
\text { CO-INTERVENTION: opioid and non-opioid } \\
\text { analgesics, adjuvant analgesics and previously directed } \\
\text { exercise program prior to enrollment } \\
\text { Duration of treatment: individually determined by } \\
\text { response of participant } \\
\text { Duration of follow-up: } 24 \text { months }\end{array}$ & $\begin{array}{l}\text { PAIN INTENSITY (NPRS, } 0 \text { to } 10 \text { scale) } \\
\text { Baseline mean: Steroid:8.2 Non-steroid: } 8.2 \\
\text { End of study mean ( } 24 \text { months): Steroid:3.2 Non- } \\
\text { steroid:3.5 } \\
\text { Reported Results: No statistically significant } \\
\text { difference between groups } \\
\text { SMD at } 3 \text { months }-0.10 \text { ( } 95 \% \text { CI Random:- } 0.46 \text { to } \\
0.25 \text { ) } \\
\text { SMD at } 6 \text { months }-0.22 \text { ( } 95 \% \text { CI Random: }-0.57 \text { to } \\
0.14 \text { ) } \\
\text { SMD at } 12 \text { months }-0.28 \text { ( } 95 \% \text { CI Random - } 0.64 \text { to } \\
0.08 \text { ) } \\
\text { FUNCTION (NDI, score of } 0 \text { to } 50 \text { with higher } \\
\text { scores indicating greater disability) } \\
\text { Baseline mean: Steroid:25.1 Non-steroid: } 25.4 \\
\text { End of study mean ( } 24 \text { months): Steroid: } 11.0 \\
\text { Non-steroid: } 11.6 \\
\text { Reported Results: No statistically significant } \\
\text { difference between groups } \\
\text { SMD at } 3 \text { months } 0.04 \text { ( } 95 \% \text { CI Random: }-0.32 \text { to } \\
0.40 \text { ) } \\
\text { SMD at } 6 \text { months -0.08 ( } 95 \% \text { CI Random: }-0.44 \text { to } \\
0.28 \text { ) } \\
\text { SMD at } 12 \text { months } 0.00 \text { ( } 95 \% \text { CI Random: }-0.36 \text { to } \\
0.36 \text { ) } \\
\text { SIDE EFFECTS: no adverse effects } \\
\text { COST OF CARE: NR }\end{array}$ \\
\hline $\begin{array}{l}\text { Manchikanti 2010b [38] } \\
\text { Method: } \\
\text { RCT } \\
\text { N(A/R): 70/70 } \\
\text { Power Analysis: done } \\
\text { Intention-to-treat Analysis: done } \\
\text { Participants: } \\
\text { Chronic discogenic neck pain w/o radiculitis w/o } \\
\text { disc herniation }\end{array}$ & $\begin{array}{l}\text { INDEX TREATMENT } \\
\text { Cervical interlaminar epidural with local anaesthetics } \\
\text { and steroids (steroid group); timing and frequency = } \\
\text { first injection at start of study and repeated based on } \\
\text { response of individual; dose }=4 \mathrm{ml} \text { lidocaine } \\
\text { hydrochloride } 0.5 \% \text { preservative free mixed with } 6 \\
\text { mg of non-particulate betamethasone; route }= \\
\text { epidural space under fluoroscopic visualization } \\
\text { (between C7 and T1 to C5 and C6) with } \\
\text { confirmation by injection of non-ionic contrast }\end{array}$ & $\begin{array}{l}\text { PAIN INTENSITY (NPRS } 0 \text { to } 10 \text { scale) } \\
\text { Baseline mean: Steroid:7.4 Non-steroid:7.8 } \\
\text { End of study mean: Steroid:3.2 Non-steroid: } 3.5 \\
\text { Reported Results: No statistically significant } \\
\text { difference between groups } \\
\text { SMD at } 3 \text { months }-0.24 \text { ( } 95 \% \text { CI Random: }-0.71 \text { to } \\
0.23 \text { ) } \\
\text { SMD at } 6 \text { months }-0.23 \text { (95\% CI Random: }-0.70 \text { to } \\
0.24 \text { ) } \\
\text { SMD at } 12 \text { months }-0.25 \text { (95\% CI Random }-0.72 \text { to } \\
0.22 \text { ) }\end{array}$ \\
\hline
\end{tabular}




\begin{tabular}{|c|c|c|}
\hline Author/Method/Participants & Intervention & Outcomes/Notes \\
\hline & $\begin{array}{l}\text { COMPARISON TREATMENT } \\
\text { cervical interlaminar epidural with local anaesthetics } \\
\text { (non-steroid group); timing and frequency = first } \\
\text { injection at start of study and repeated based on response } \\
\text { of individual; dose }=5 \mathrm{ml} \text { of lidocaine hydrochloride } \\
0.5 \% \text { preservative free; route = epidural space under } \\
\text { fluoroscopic visualization (between C7 and T1 to C5 and } \\
\text { C6) with confirmation by injection of non-ionic contrast } \\
\text { CO-INTERVENTION: opioid and non-opioid } \\
\text { analgesics, adjuvant analgesics and previously directed } \\
\text { exercise program prior to enrollment, medication based } \\
\text { on medical necessity } \\
\begin{array}{l}\text { Duration of treatment: at baseline, another round done } \\
\text { only if response to injection was positive in physical and } \\
\text { only when increased levels of pain reported with } \\
\text { deteriorating relief below } 50 \% \\
\text { Duration of follow-up: } 12 \text { months }\end{array}\end{array}$ & $\begin{array}{l}\text { FUNCTION (NDI, score of } 0 \text { to } 50 \text { with higher scores } \\
\text { indicating greater disability) } \\
\text { Baseline mean: Steroid:28.5 Non-steroid:30.0 } \\
\text { End of study mean: Steroid:12.7 Non-steroid: } 14.4 \\
\text { Reported Results: No statistically significant } \\
\text { difference between groups } \\
\text { SMD at } 3 \text { months }-0.24 \text { ( } 95 \% \text { CI Random }-0.71 \text { to } \\
0.23 \text { ) } \\
\text { SMD at } 6 \text { months }-0.25 \text { (95\% CI Random: }-0.72 \text { to } \\
0.22 \text { ) } \\
\text { SMD at } 12 \text { months }-0.25 \text { (95\% CI Random }-0.72 \text { to } \\
0.22 \text { ) } \\
\text { SIDE EFFECTS: nerve root irritation reported in } 3 \\
\text { patients w/o long-term sequelae, gave them } 8 \text { mg of } \\
\text { decadron intravenously } \\
\text { COST OF CARE: NR }\end{array}$ \\
\hline $\begin{array}{l}\text { Naja 2006 [35] } \\
\text { Method: } \\
\text { RCT } \\
\text { N(A/R): } 47 / 50 \\
\text { Power Analysis: done } \\
\text { Intention-to-treat Analysis: not done } \\
\text { Participants: } \\
\text { Chronic cervicogenic headache }\end{array}$ & $\begin{array}{l}\text { INDEX TREATMENTS } \\
\text { Either greater occipital nerve and lesser occipital nerve } \\
\text { anesthetic block OR greater occipital nerve and lesser } \\
\text { occipital nerve with facial nerve blockade depending on } \\
\text { the extension of the headache; timing and frequency = } \\
\text { one treatment after } 2 \text { weeks of baseline assessment; dose } \\
=3 \text { ml anesthetic mixture; route = nerve stimulator guided } \\
\text { nerve block } \\
\text { COMPARISON TREATMENT } \\
\text { Preservative free normal saline injection; timing and } \\
\text { frequency = one treatment after } 2 \text { weeks of baseline } \\
\text { assessment; dose = 3ml normal saline; route = nerve } \\
\text { stimulator guided nerve block } \\
\text { CO-INTERVENTION: analgesic consumption: for VAS } \\
<4 \text { oral paracetamol 500mg maximum } 6 \text { tablets/day Or } \\
\text { ketoprofen } 100 \mathrm{mg} \text { maximum } 3 \text { tablets/day; for VAS > } 4 \\
\text { oral combo of dextropropoxyphene } 30 \mathrm{mg} \text { and } \\
\text { paracetamol } 400 \mathrm{mg} \text { maximum } 6 \text { tablets/day Or tramadol } \\
\text { hydrochloride 100mg tablets maximum } 3 \text { tablets/day } \\
\text { Duration of treatment: One treatment } \\
\text { Duration of follow-up: } 2 \text { weeks }\end{array}$ & $\begin{array}{l}\text { PAIN (VAS } 0 \text { to } 10 \mathrm{~cm} \text { ) } \\
\text { Baseline Mean: anesthetic block 6.26, normal saline } \\
6.28 \\
\text { End of study Mean: anesthetic block 4.42, normal } \\
\text { saline } 6.35 \\
\text { Reported Results: significant difference between } \\
\text { anesthetic block group and normal saline group } \\
\text { SMD at } 2 \text { weeks follow up } 1.16 \text { (95\% CI Random: - } \\
1.78 \text { to -0.54) } \\
\text { PAIN (Total Pain Index) } \\
\text { Baseline Mean: anesthetic block 358.68, normal saline } \\
\text { 352.46 } \\
\text { End of study Mean: anesthetic block 194.25, normal } \\
\text { saline } 329.96 \\
\text { Reported Results: significant difference between } \\
\text { anesthetic block group and normal saline group } \\
\text { SIDE EFFECTS: NR } \\
\text { COST OF CARE: NR }\end{array}$ \\
\hline $\begin{array}{l}\text { Sand 1992 [40] } \\
\text { Method: } \\
\text { RCT (crossover) } \\
\text { N(A/R): } 20 / 20 \\
\text { Power Analysis: NR } \\
\text { Intention-to-treat Analysis: NR } \\
\text { Participants: } \\
\text { Neck disorder with headache, duration of disorder } \\
\text { NR }\end{array}$ & $\begin{array}{l}\text { INDEX TREATMENT } \\
\text { Sterile Water: timing and frequency }=5 \text { to } 27 \text { cervical } \\
\text { trigger points injected, dose }=0.3 \mathrm{ml} \text {, duration }=1 \text { session } \\
\text { single treatment, route }=\text { intracutaneous } \\
\text { COMPARISON TREATMENT } \\
\text { Isotonic Saline: timing and frequency }=5 \text { to } 27 \text { cervical } \\
\text { trigger points injected, dose }=0.3 \mathrm{ml} 0.9 \% \text { normal saline, } \\
\text { duration }=1 \text { session single treatment, route }= \\
\text { intracutaneous } \\
\text { CO-INTERVENTION: NR } \\
\text { Duration of treatment: } 1 \text { session } \\
\text { Duration of follow-up: } 13 \text { days }\end{array}$ & $\begin{array}{l}\text { PAIN INTENSITY (VAS 0 to 100) } \\
\text { Baseline: NR } \\
\text { Reported Results: not significant } \\
\text { SMD: }-0.09 \text { (95\% CI Random: }-0.96 \text { to } 0.79 \text { ) (power } \\
6 \% \text { ) } \\
\text { AROM (mean three planes, degrees) } \\
\text { Baseline: NR } \\
\text { Reported Results: not significant } \\
\text { SMD: } 0.35(95 \% \text { CI Random: }-0.53 \text { to } 1.23 \text { ) } \\
\\
\text { SIDE EFFECT: at the end of } 12 \text { hours slight increase } \\
\text { mean pain level for both treatments } \\
\text { COST OF CARE: NR }\end{array}$ \\
\hline $\begin{array}{l}\text { Terzi 2002 [36] } \\
\text { Method: } \\
\text { RCT } \\
\text { N(A/R): 20/20 } \\
\text { Power Analysis: NR } \\
\text { Intention-to-treat Analysis: NR } \\
\text { Participants: } \\
\text { Cervicogenic headache, duration of disorder NR }\end{array}$ & $\begin{array}{l}\text { INDEX TREATMENT } \\
\text { Prilocaine: timing = } 1 \text { injection; frequency }=\text { once; dose = } \\
1 \mathrm{ml} \text { of } 2 \% \text { solution; duration }=30 \text { minutes; route }= \\
\text { injection of greater occipital nerve } \\
\text { COMPARISON TREATMENT } \\
\text { Placebo: normal saline } 1 \text { ml into greater occipital nerve } \\
\text { CO-INTERVENTION: NR } \\
\text { Duration of treatment: } 1 \text { session } \\
\text { Duration of follow-up: } 30 \text { minutes }\end{array}$ & $\begin{array}{l}\text { PAIN INTENSITY (VAS 0 to 10) } \\
\text { Baseline: prolocaine } 6.6 \text {, saline } 7.2 \\
\text { End of Study Mean: prolocaine } 1.7 \text {, saline } 6.6 \\
\text { Absolute Benefit: prolocaine } 4.9 \text {, saline } 0.6 \\
\text { Reported Results: significant, favouring prolocaine } \\
\text { SMD: -3.60 (95\% CI Random: }-5.12 \text { to }-2.07 \text { ) } \\
\text { SIDE EFFECTS: NR } \\
\text { COST OF CARE: NR }\end{array}$ \\
\hline
\end{tabular}

Key: AROM - active range of movement; BDI - Beck Depression Inventory; Botulinum toxin type A (BoNT-A); $\mathrm{CO}_{2}$ - Carbon Dioxide; cntl - control; $95 \%$ CI - 95\% confidence interval; DNG - dry needling; LiC - lidocaine; N(A/R) - total number of participant (analysed/randomized); ml - milliliter; mg - milligram; MPQ - McGill Pain Questionnaire; NA - not applicable; NPRS - Numeric Pain Rating Scale; NDI - Neck Disability Index; NR - not reported; RCT - randomized controlled trial; RR - relative risk; SCDI - subcutaneous insufflation; SMD - standard mean difference; SPGB - sphenopalatine ganglion block; US - ultrasound; VAS - Visual Analogue Scale; w/o - without. 


\section{Dealing with Missing Data}

We contacted authors for missing data.

\section{Assessment of Heterogeneity}

Prior to calculation of a pooled effect measure, we assessed clinical heterogeneity through an examination of issues such as: symptom duration (acute $v s$ chronic); subtype of neck pain (e.g. myofascial neck pain $v s$ degenerative); intervention type (e.g. local anaesthetics $v s$ corticosteroids); characteristics of treatment (e.g. dosage, technique); and outcomes (pain relief, measures of function and disability, patient satisfaction, quality of life). We pooled the studies if it was clinically sensible to do so, using random-effects models, since random-effects models are more conservative than fixed-effects meta-analysis. We assessed statistical heterogeneity using the Cochran $\mathrm{Q}$ test, by calculating $\mathrm{I}^{2}$ values (with $\mathrm{I}^{2}>50 \%$ considered to represent substantial heterogeneity) and tau squared values.

\section{Assessment of Reporting Biases}

Sensitivity analysis was used to examine for the influence of potential reporting bias on meta-analysis results.

\section{Data Synthesis}

We assessed the quality of the body of available evidence using the Grading of Recommendations Assessment, Development and Evaluation (GRADE) approach [22]. Domains that may decrease the quality of the evidence are: 1) the study design, 2) risk of bias, 3) inconsistency of results, 4) indirectness (not generalizable), 5) imprecision (insufficient data), and other factors (e.g. reporting bias). The quality of the evidence was downgraded by a level based on the performance of the studies against these five domains.

Levels of quality of evidence were defined as:

- High quality evidence: Further research is very unlikely to change our confidence in the estimate of effect. All of the GRADE domains are met.

- Moderate quality evidence: Further research is likely to have an important impact on our confidence in the estimate of effect and may change the estimate. One of the domains is not met.

- Low quality evidence: Further research is very likely to have an important impact on our confidence in the estimate of effect and is likely to change the estimate. Two of the domains are not met.

- Very low quality evidence: We are very uncertain about the estimate. Three of the domains are not met.

- $\quad$ No evidence: no RCTs were identified that measured the outcome of interest.

\section{Subgroup Analysis and Investigation of Heterogeneity}

For the majority of studies we analysed the results separately for duration of follow-up (immediately post treatment, short-term, intermediate term, and long-term) and subtypes of injection (intramuscular local anaesthetic, nerve block steroid, nerve block anaesthetic, epidural, intracutaneous neutral agent, and transforaminal steroid). We conducted a subgroup analysis of pooled studies to investigate clinical and statistical heterogeneity when necessary.

\section{Sensitivity Analysis}

Sensitivity analysis or meta-regression was considered for the following factors: symptom duration, risk of bias, and subtype of neck disorder and these were carried out when there was sufficient evidence to warrant these assessments.

\section{RESULTS}

\section{Description of Studies}

Considering all sources, 2432 records were identified through database searches and 22 records were found from other sources until March 2012. Following the screening step, there were 40 candidate publications evaluated in the selection phase (Fig. 1). Inter-rater agreement on study selection for inclusion was very good, with an estimated quadratic weighted kappa of $\mathrm{Kw}=0.79$ (SD 0.35) [19]. Three publications represented one trial [28-30].

Thus of the 12 trials included (See Characteristics of included studies (Table 2), one was conducted in acute nonspecific neck pain, nine were in chronic MND (four in myofascial pain, one in radiculopathy, one in discogenic pain, one in cervicogenic headache, one in non-specific neck pain and one in a mixed disorder population) and two were conducted in patients with cervicogenic headache, where the duration of symptoms was not reported. There was a spectrum of interventions studied in these 12 trials including:

- $\quad$ Four studies using IM local anaesthetic: [31-34].

- Four studies using nerve block: steroid [28-30], anaesthetic [35-37].

- $\quad$ One study using epidural steroid: [38].

- Two studies using intracutaneous neutral agents such as carbon dioxide insufflation and saline respectively: $[39,40]$.

- $\quad$ One study using transforaminal steroid: [41].

We excluded 26 RCTs [42-67] that did not apply interventions of interest $(54 \%$; 14/26) (i.e. oral medication, botox, exercise or intravenous agents) or they used an inappropriate comparator $(46 \%$; 12/26) (See Characteristics of excluded studies - APPENDIX B).

\section{Risk of Bias in Included Studies}

We found four of the 12 trials had a low risk of bias [28$30,35,36,41]$ and 8 trials had high risk of bias [31-34,37-40]. See Fig. (2) for a summary graph of risk of bias assessments.

Regarding selection bias, we found that $67 \%$ (8/12) of the trials did not describe or use appropriate randomization methods and $83 \%(10 / 12)$ did not conceal allocation. The lack of both effective blinding for patients in 50\% (6/12) and for the provider in $58 \%(7 / 12)$ of the trials contributed to performance bias. We found detection bias in 50\% (6/12) of the trials due to lack of blinding of the outcome assessor. The drop-out rate was not reported and use of an intention to treat analysis was not described or inadequate in 58\% (7/12) of the trials, contributing to an attrition bias. We found that the similarity of baseline characteristics in the most 


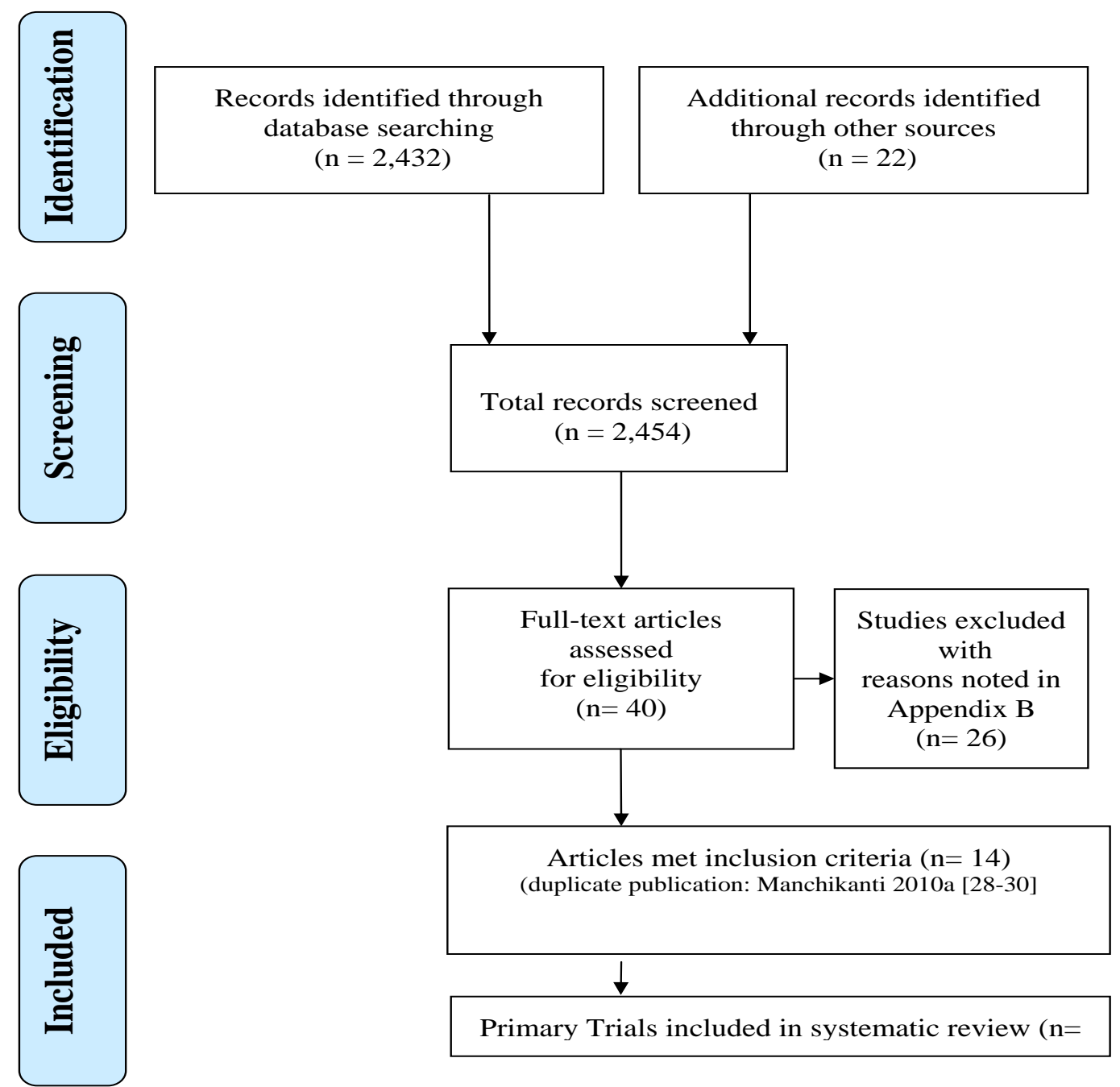

Fig. (1). PRISMA diagram showing the flow of reviews.

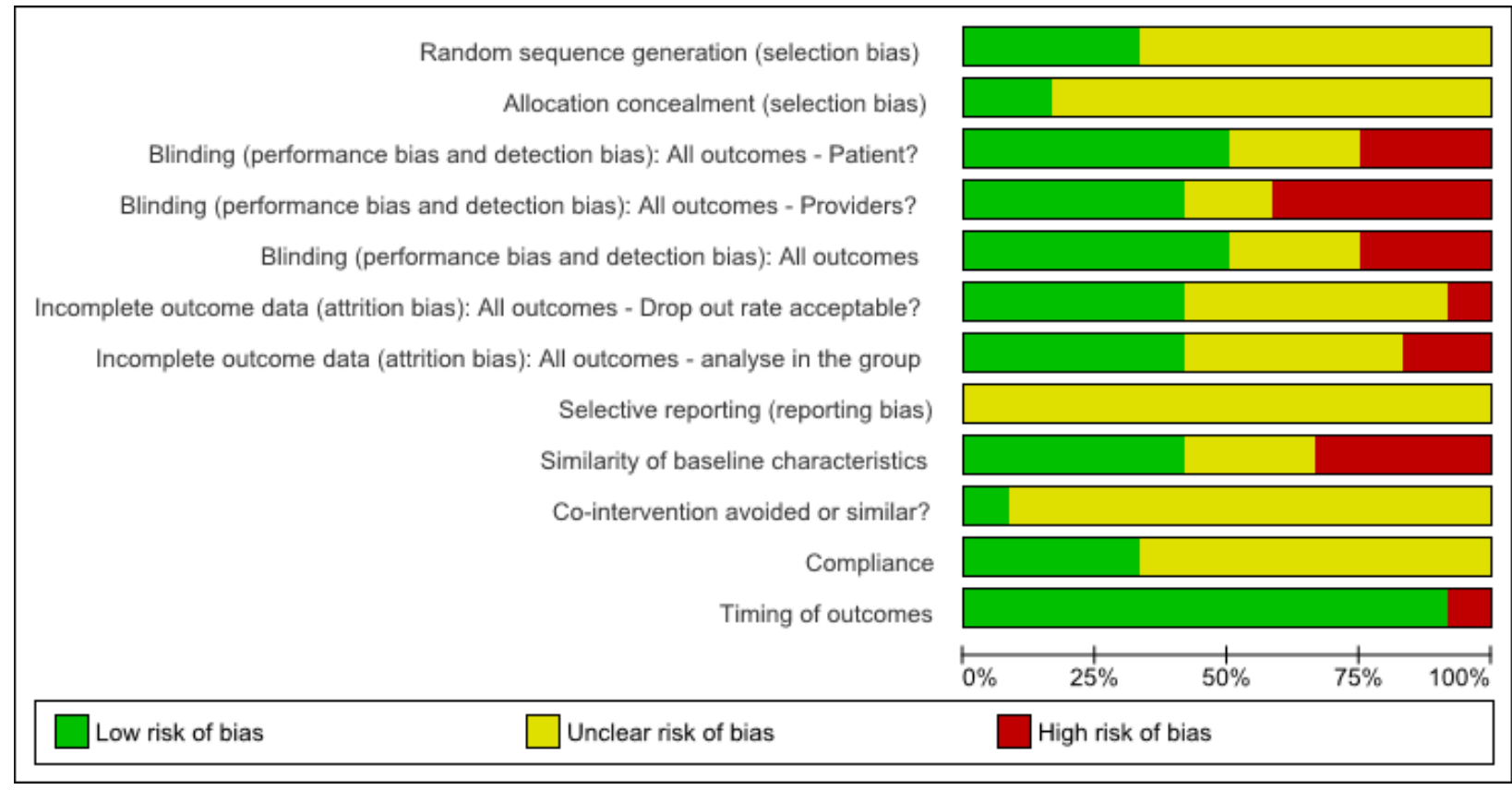

Fig. (2). Risk of bias graph. 
important prognostic indicators was also inadequate in $58 \%$ (7/12) of the included trials. We determined that cointerventions were not similar or not avoided in $92 \%(11 / 12)$ of trials and compliance to the intervention was unclear in $67 \%(8 / 12)$ of trials included. The timing of outcomes was similar in all but one trial, with the duration of follow-up assessment varying from 5 minutes post intervention to 24 months post. We determined that selective reporting was a high risk of bias in all studies as this item requires the authors to report the results for all outcome measures to be used in the study priori. Selective reporting can be problematic to assess as the published results need to be compared to their study protocol, with most studies not having adequate documentation of their study protocol. We explored publication bias for the meta-analysis intramuscular injection (local anaethetic) in Fig. (4) using a Funnel Plot and could not rule publication bias. In other words, the asymmetrical plot suggests the smaller trials [32,34] of lower methodological quality produced exaggerated intervention effect estimates.

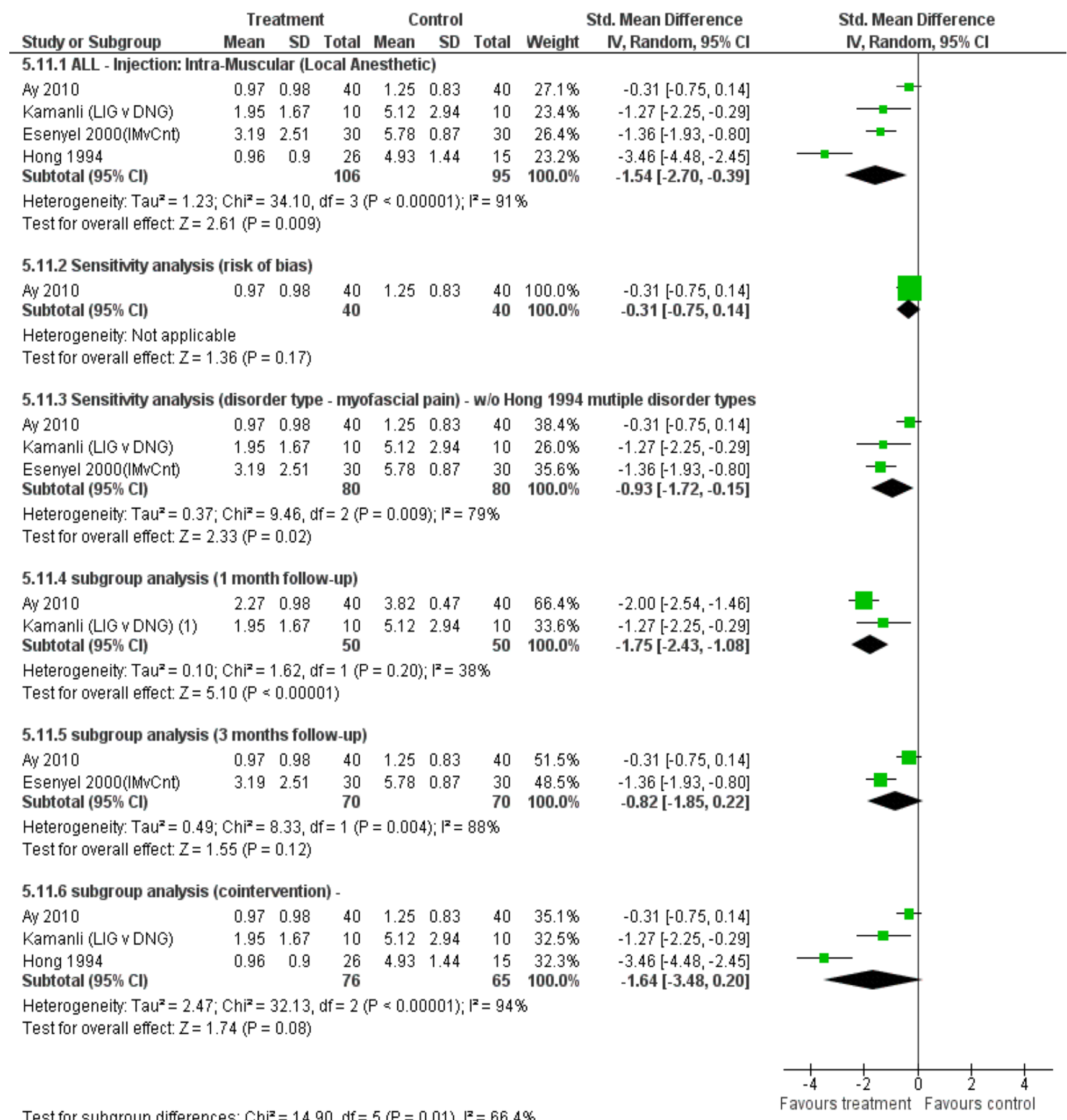

Test for subgroup differences: $\mathrm{Chi}^{2}=14.90, \mathrm{df}=5(\mathrm{P}=0.01), \mathrm{I}^{\mathbf{2}}=66.4 \%$

Favours treatment Favours control

Fig. (3). Forest plot investigation of heterogeneity. 


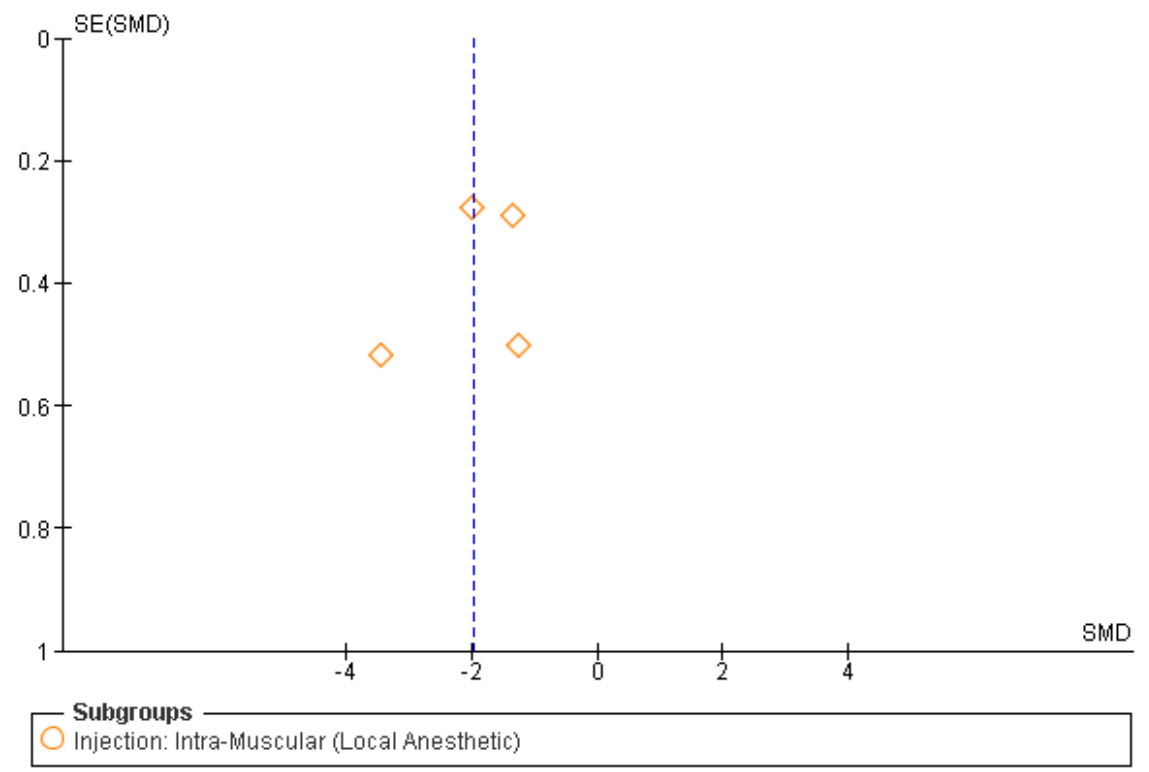

Fig. (4). Funnel plot of comparison: for meta-analysis Intramuscular injection (local anaethetic - lidocaine) vs Control for outcome: pain intensity - short term.

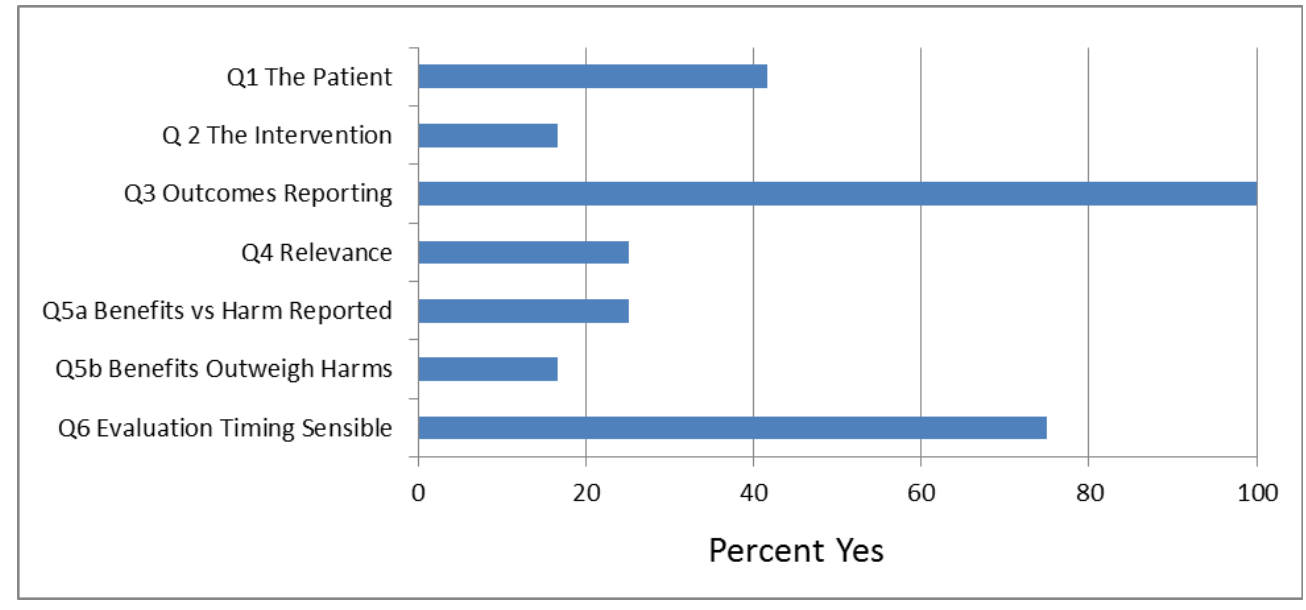

Fig. (5). Clinical applicability and relevance.

In summary, we found the risk of bias to be high in the majority of studies. Five of the 12 trials appeared to be fatally flawed as they lacked reporting on almost all items in the Cochrane risk of bias criteria.

\section{Effects of Interventions}

We present our data in the summary of findings table (Table 3).

\section{Evidence of Benefit}

\section{High or Moderate Quality Evidence}

There were no physician-delivered injections that met the criteria for high or moderate quality evidence of benefit.

\section{Low or Very Low Quality of Evidence}

Intramuscular lidocaine injection with or without neck stretches vs dry needling or placebo
We found limited evidence that there may be benefit in the short-term with use of IM-lidocaine with or without stretching $v s$ dry needling or placebo (4 trials [31-34] with 201 participants for pain with a pooled effect size of SMD 1.54 (95\% CI -2.70 to -0.39$)$ ). These 4 trials were pooled because they were clinically similar in terms of the type of injection, location of injection and the patient population was chronic MNDs. However, the pooled results show considerable statistical heterogeneity $\left(\mathrm{Tau}^{2}=1.23 ; \mathrm{Chi}^{2}=\right.$ $\left.34.10, \mathrm{df}=3(\mathrm{P}<0.00001) ; \mathrm{I}^{2}=91 \%\right)$. We explored sources of this heterogeneity arising from risk of bias, subtype disorder, duration of follow-up and co-intervention.

The reader is referred to Fig. (3) for a forest plot of different comparisons of subgroup and sensitivity analyses. After removal of the fatally flawed trials, the one remaining trial [31] showed a dramatic decrease in the treatment effect from SMD - 1.54 (95\% CI -2.70 to -0.39$)$ in the pooled effect to SMD -0.31 (95\% CI -0.75 to 0.14 ) for 
Table 3. Summary of Findings Table

\begin{tabular}{|c|c|c|c|c|c|c|c|c|c|}
\hline & & & & & & \multicolumn{4}{|c|}{ Summary of Findings } \\
\hline \multicolumn{6}{|c|}{ Quality Assessment } & \multicolumn{2}{|c|}{$\begin{array}{l}\text { Number of } \\
\text { Participants }\end{array}$} & \multirow[b]{2}{*}{ 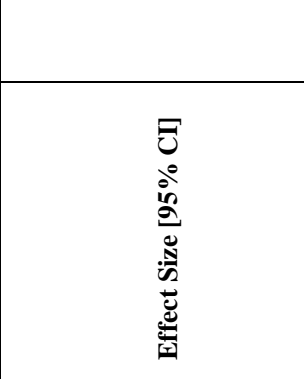 } & \multirow[t]{2}{*}{$\begin{array}{c}\text { Quality } \\
\text { (GRADE) }\end{array}$} \\
\hline 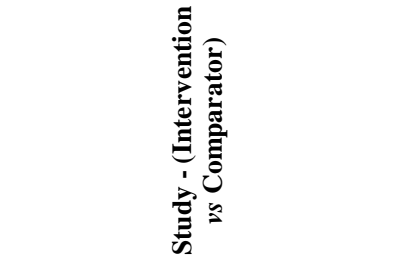 & 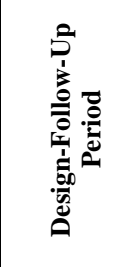 & 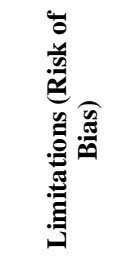 & 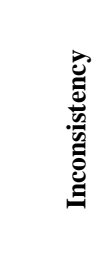 & 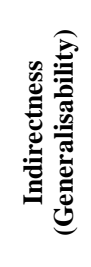 & 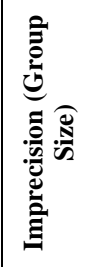 & $\stackrel{=}{. \pm}$ & 巳e & & \\
\hline \multicolumn{10}{|l|}{ Evidence of benefit } \\
\hline \multicolumn{10}{|c|}{ 1. Injection: Intra-Muscular (Local Anaesthetic) } \\
\hline Kamanli 2005 [32] (LD vs DNG) & $\begin{array}{l}\text { RCT-ST } \\
(1 \mathrm{mo})\end{array}$ & High $(-1)$ & N/A & N/A & -1 & 10 & 10 & SMD -1.27 [-2.25, -0.29] & Very Low \\
\hline $\begin{array}{l}\text { Esenyel } 2000[33](\mathrm{LD}+ \\
\text { stretches } v s \text { stretches) }\end{array}$ & $\begin{array}{l}\text { RCT-ST } \\
(3 \text { mos })\end{array}$ & High (-1) & N/A & N/A & -1 & 30 & 30 & SMD $-1.36[-1.93,-0.80]$ & Very Low \\
\hline Hong 1994 [34] (LD vs DNG) & $\begin{array}{l}\text { RCT-ST } \\
\text { (2 wks) }\end{array}$ & High $(-1)$ & N/A & N/A & -1 & 26 & 15 & SMD -3.46 [-4.48, -2.45] & Very Low \\
\hline \multicolumn{10}{|l|}{ FUNCTION and DISABILITY } \\
\hline Kamanli 2005 [32] (LD vs DNG) & $\begin{array}{l}\text { RCT-ST } \\
(1 \mathrm{mo})\end{array}$ & $\operatorname{High}(-1)$ & N/A & N/A & -1 & 10 & 10 & SMD -1.05 $[-2.00,-0.10]$ & Very Low \\
\hline \multicolumn{10}{|l|}{ QUALITY OF LIFE } \\
\hline $\begin{array}{l}\text { Naja } 2006 \text { [35] (GON + LON +/- } \\
\text { facial: LD vs saline) }\end{array}$ & RCT-ST & Low $(0)$ & N/A & N/A & -1 & 24 & 23 & SMD -1.16 [-1.78, -0.54] & Low \\
\hline \multicolumn{10}{|c|}{ Evidence of NO benefit ( $v s$ control) or no difference ( $v s$ active comparison) } \\
\hline \multicolumn{10}{|c|}{ 3. Injection: Nerve Block (Steriod + anaesthetic $v s$ anaesthetic) } \\
\hline \multicolumn{10}{|l|}{ PAIN } \\
\hline \multirow{3}{*}{$\begin{array}{l}\text { Manchikanti } 2010 \text { [28- } \\
\text { 30](medial brangh block - } \\
\text { Steriod + Bupivacaine } v s \\
\text { Bupivacaine) }\end{array}$} & RCT-ST & \multirow[t]{3}{*}{ Low $(0)$} & \multirow[t]{3}{*}{ N/A } & \multirow[t]{3}{*}{ N/A } & \multirow[t]{3}{*}{-1} & \multirow[t]{3}{*}{60} & \multirow[t]{3}{*}{60} & SMD $-0.10[-0.46,0.25]$ & \multirow[t]{3}{*}{ Moderate } \\
\hline & RCT-IT & & & & & & & SMD $-0.22[-0.57,0.14]$ & \\
\hline & RCT-LT & & & & & & & SMD $-0.28[-0.64,0.08]$ & \\
\hline \multicolumn{10}{|l|}{ FUNCTION AND DISABILITY } \\
\hline \multirow[t]{3}{*}{ as above } & RCT-ST & & & & & & & SMD $0.04[-0.32,0.40]$ & \\
\hline & RCT-IT & & & & & & & SMD $-0.08[-0.44,0.28]$ & \\
\hline & RCT-LT & & & & & & & SMD $0.00[-0.36,0.36]$ & \\
\hline \multicolumn{10}{|c|}{ 4. Injection: Intra-Muscular (Local Anaesthetic) } \\
\hline \multicolumn{10}{|l|}{ PAIN } \\
\hline Ay 2010 [31] (LD vs DNG) & $\begin{array}{l}\text { RCT-ST } \\
(3 \text { mos })\end{array}$ & $\operatorname{High}(-1)$ & N/A & N/A & -1 & 40 & 40 & SMD $-0.31[-0.75,0.14]$ & Low \\
\hline
\end{tabular}




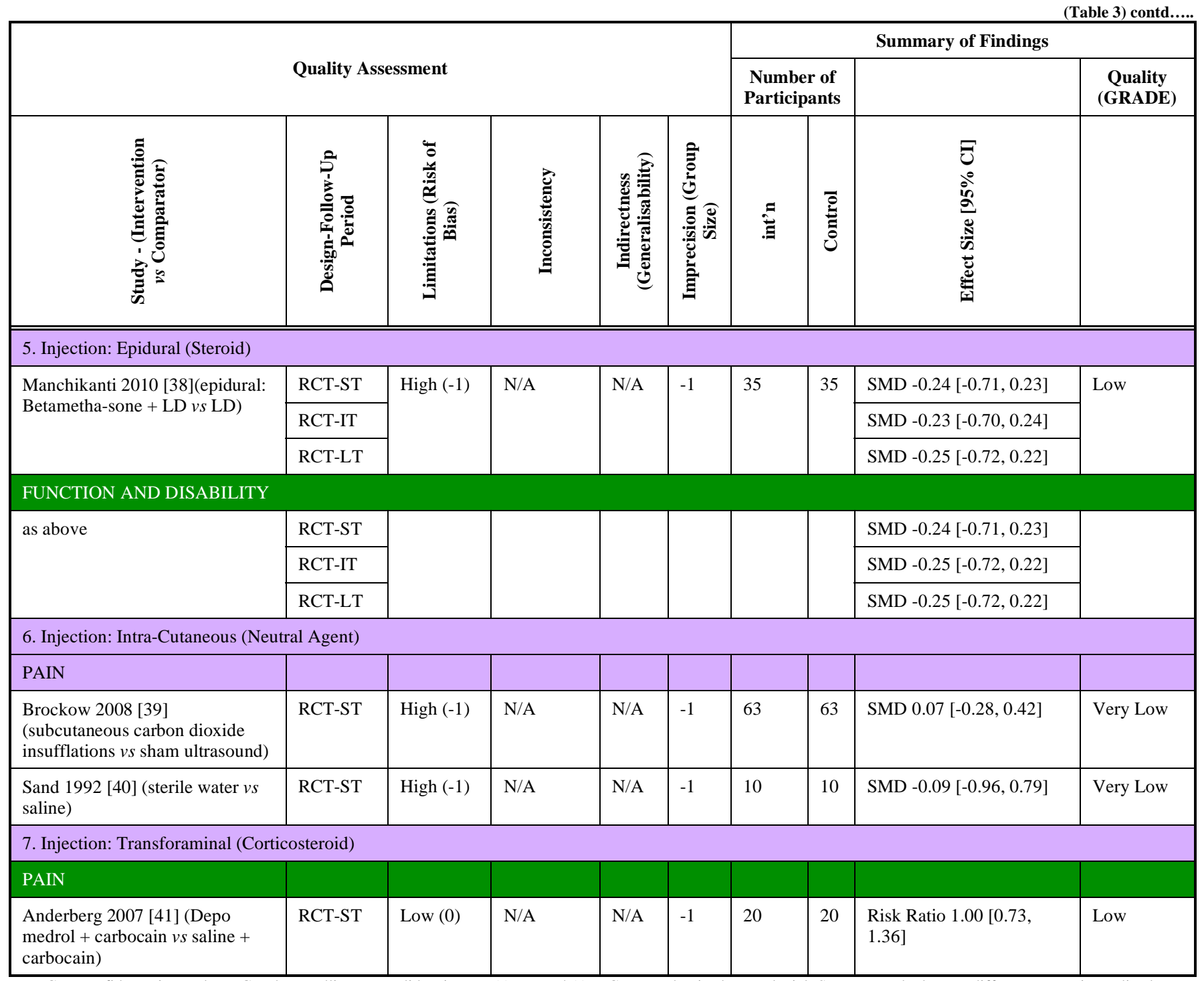

Key: CI - confidence interval, DNG - dry needling, LD - lidocaine, mo(s) - month(s), RCT - randomized control trial, SMD - standard mean difference, IP - immediately post treatment (within 1 day), ST - short-term (closest to 4 weeks), IT - intermediate term (closest to 6 months), LT - long-term (closest to 12 months).

the single study. When we also removed Hong 1994 [34] since it included mixed disorder type participants and the other studies only looked at myofascial pain, this also resulted in a major decrease in magnitude of effect. Furthermore, a difference was seen favouring intervention when shorter term follow-up of 1 month studies (SMD $1.75(95 \%$ CI -2.43 to -1.08$)$ ) were compared to the 3 month follow-up studies (SMD $-0.82(95 \%$ CI $\quad-1.85$ to $0.22)$ ). Finally as Esenyel 2000 [33] was the one study to include neck stretching exercises in combination with injection, we examined the effect of removing this study. Doing so resulted in no major change in the pooled effect.

Based on very low quality evidence (1 trial [32]: 20 participants) there may be a benefit with the use of IMlidocaine over dry needling on function and disability (SMD $-1.05(95 \%$ CI -2.00 to -0.10$)$ ) and quality of life (SMD 1.24 (95\% CI -2.22 to -0.27)) over short-term follow-up.

\section{Nerve Block Anaesthetic vs Control}

We found low quality evidence (1 trial [36]; 20 participants) that there was a statistically significant improvement immediately post treatment for pain (SMD -
$3.60(95 \%$ CI -5.12 to -2.07$))$ in a patient population with cervicogenic headache between those receiving greater occipital nerve blockade with prilocaine $v s$ those receiving greater occipital nerve blockade with normal saline injection.

Low quality evidence from one trial [35] (47 participants) found a statistically significant improvement for pain [SMD -1.16 (95\% CI -1.78 to -0.54)] in the short-term for patients with cervicogenic headache between those patients receiving occipital nerve blockade with lidocaine $v s$ those receiving saline.

No other physician delivered injections were found to show evidence of benefit for pain, function and disability, quality of life, global perceived effect and patient satisfaction.

\section{Evidence of No Benefit (vs Control) or No Difference (vs Active Comparison)}

\section{Moderate Quality of Evidence}

\section{Nerve Block: Steroid}

We found moderate quality evidence (1 trial [28-30]; 120 participants) reporting no difference using a medial branch 
block with betamethasone and bupivacaine $v s$ bupivacaine alone at short term (SMD -0.10 (95\% CI -0.46 to 0.25$)$ ), intermediate term (SMD -0.22 (95\% CI -0.57 to 0.14$)$ ) and long-term follow up (SMD -0.28 (95\% CI -0.64 to 0.08)) for pain in patients with chronic non-specific neck pain. Further this trial reported no evidence of benefit for function and disability as well at short term (SMD 0.04 (95\% CI -0.32 to $0.40)$ ), intermediate term (SMD -0.08 (95\% CI -0.44 to $0.28)$ ) and long-term follow up (SMD 0.00 (95\% CI -0.36 to 0.36)). For long term follow up, we have presented the data from 12 month follow-up alone because it does not differ importantly from the data reported at 2 year follow-up.

\section{Low or Very Low Quality of Evidence}

\section{Intramuscular Lidocaine Injection vs Dry Needling}

We found low quality evidence (1 trial [31]; 80 participants) that reported outcomes on pain at both 4 weeks (SMD -2.00 (95\% CI -2.54 to -1.46)) and 12 weeks (SMD $0.31(95 \% \mathrm{CI}-0.75$ to 0.14$)$ ). At 4 weeks there was evidence of benefit for IM-lidocaine $v s$ dry needling; however, at 12 weeks there was no evidence of benefit of the injection over the control.

\section{Epidural: Steroid}

We found low quality evidence (1 trial [38]; 70 participants) that showed there was no difference in effect of betamethasone and lidocaine injected into the epidural space as compared to lidocaine alone, as assessed by pain shortterm (SMD -0.24 (95\% CI -0.71 to 0.23)), intermediate-term (SMD -0.23 (95\% CI -0.70 to 0.24)), and long-term (SMD 0.25 (95\% CI -0.72 to 0.22$)$ ). Further there was no evidence of additional benefit for function and disability over the short-term (SMD $-0.24 \quad(95 \% \quad$ CI -0.71 to 0.23$)$ ), intermediate-term (SMD -0.25 (95\% CI -0.72 to 0.22)), and long-term (SMD -0.25 (95\% CI -0.72 to 0.22)).

Intracutaneous Neutral Agents vs Control

There was very low quality evidence from two studies that looked at intracutaneous neutral agents: carbon dioxide vs sham ultrasound (1 trial [39]; 126 participants) and water $v s$ saline (1 trial [40]; 20 participants) in the short-term. Both studies showed there was no significant difference in effects from these agents $v s$ the controls for pain in acute nonspecific neck pain [39] or neck disorder with headache [40].

\section{$\underline{\text { Transforaminal: Corticosteroid }}$}

We found low quality evidence from one study comparing depo medrol and carbocain vs carbocain alone ([41]; 40 participants) that showed there was no difference in outcomes of pain (RR 1.00 (95\% CI 0.73 to 1.36$)$ in the short-term between the two groups.

\section{Nerve Block: Anaesthetic}

We found very low quality evidence (1 trial [37]; 23 participants) of no significant difference in pain intensity between sphenopalatine ganglion block with lidocaine $v s$ placebo in the short-term; however we were unable to extract data to confirm their data analysis.

\section{Clinical Applicability and Relevance}

We used three criteria to assess clinical applicability to practice (descriptions of the patient, interventions, and outcomes) and three to assess the clinical relevance of the results (relevance, benefits $v s$ harms, and timing of evaluation). See Fig. (5) for a summary graph of clinical applicability assessments.

We found that $42 \%(5 / 12)$ of included studies described the patient study population sufficiently. Only $17 \%(2 / 12)$ of studies provided adequate detail for the intervention protocol to allow the intervention to be replicated. These two studies [28-31] adequately described the skills, training and experience of the person who delivered the injection. All studies evaluated the effect of the treatment on pain, and three studies [28-30,32,38] also evaluated the outcome of function and disability. We found that $75 \%$ (9/12) of studies used appropriate timing for the evaluation of outcomes taking into consideration the drugs mechanism of action and expected treatment duration. Twenty-five percent (3/12) of studies adequately reported the rate and severity of adverse events, the adherence to treatment and the drop-out rate. Only two studies $[32,34]$ demonstrated a clinically important difference in pain intensity between the treatment and the control groups. In $17 \%(2 / 12)$ of studies we concluded that the treatment benefits were worth the potential harms of the injection.

\section{Adverse Events}

Fifty-eight percent (7/12) of studies reported on adverse events. All adverse events described were transient and benign. Adverse events included: nerve root irritation, slight increase in mean pain levels, allergic reaction, increase in radicular pain, or the presence of pain, burning or paraesthesia at the injection site. See Table 4 for specific adverse events reported for each injection type.

\section{DISCUSSION}

The conclusions from this systematic review update are limited due to the limited nature of the studies included. We found a high risk of bias, a low quality of evidence and limited clinical applicability of the results from these trials. Higher quality studies showing evidence of benefit are needed in order to be confident in the use of physiciandelivered injections for MND and that these potential benefits exceed their risks. Further studies are warranted.

\section{Summary of Main Results}

In this update, we found no high or moderate quality evidence demonstrating the benefits of medicinal injections to treat MNDs. One low quality trial showed benefit for IMlidocaine injection for chronic mechanical neck pain at 4 weeks but no persistence of the benefit at 12 weeks. We also found low and very low quality evidence for IM-lidocaine injection for chronic mechanical neck pain and nerve block anaesthetic for cervicogenic headache.

Only one moderate quality study was found and it showed that there was no difference between steroids and controls for mechanical neck pain. All other trials were low or very low quality and showed no benefit of injection over controls or placebo.

\section{Overall Completeness and Applicability of Evidence}

We found that the applicability of these trials to practice was limited. While all trials looked at the effect of medicinal 


\section{Table 4. Adverse Events}

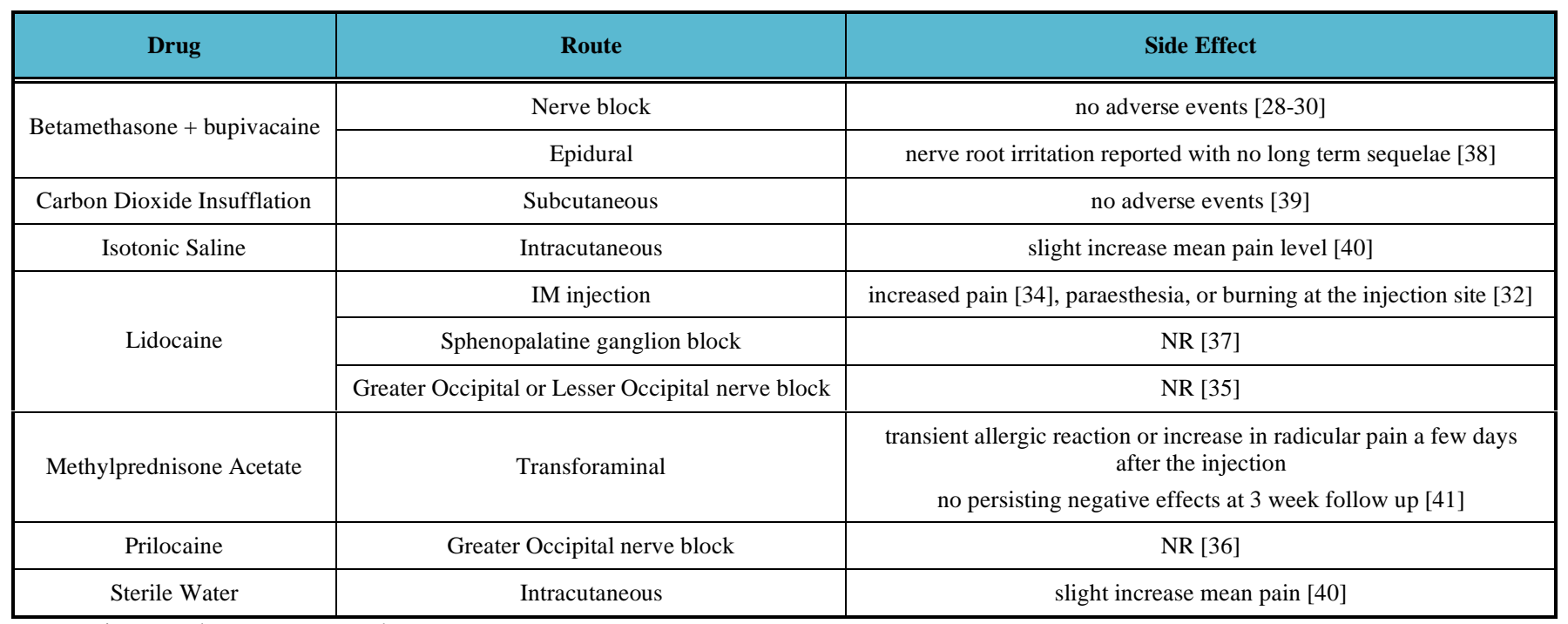

injections on pain and a majority used appropriate timing of follow-up, many trials did not adequately describe the patient population or the intervention. The application of the noted treatments identified to be effective for mechanical neck pain may extend to other neck pain classification systems such as insidious onset of neck pain, traumatic neck pain, neck pain with headache. The concordance between neck pain classification systems is unclear and the clinical applicability will need to be based on sound clinical judgment. Few reported adequately on the severity of adverse events.

\section{Quality of the Evidence}

No conclusion regarding the effectiveness of medicinal injections for adults with MNDs could be made because there was a complete lack of high quality evidence supporting their use. There was only one moderate quality study ([28-30]; 120 participants) which had a low risk of bias, long-term follow-up (2 years) and a moderate sample size. The remainder of the studies were either low or very low quality. There was large heterogeneity across studies and therefore it was difficult to pool data to provide summary estimates of effects. Many of the studies reported here had small sample sizes and were conducted in a single centre, which limits their quality (GRADE) of the evidence.

\section{Potential Biases in the Review Process}

There may be language bias in our review because we did not explicitly search non-English databases; however, we did not exclude studies that weren't published in English. We did not search the grey literature, such as, searching databases for unpublished work, writing to authors for additional unpublished data and contacting agencies and this review likely has a bias towards the published literature. We attempted to minimize this bias by employing a research librarian to complete very systematic bibliographic searches. We attempted to minimize selection bias by the use of two independent reviewers from different professional backgrounds to consider selection.

\section{Agreements and Disagreements with Other Studies or Reviews}

In the ICON review [16] the authors found low quality evidence to support the use of IM-lidocaine for chronic MND, and epidural methylprednisolone with lidocaine injection for radiculopathy. There was a lack of evidence for the effectiveness of different types of corticosteroids and local anaesthetics. The authors also concluded that larger high quality trials were needed to support the use of any of these medicinal injections.

Since the search strategy conducted to inform the ICON review was conducted, we found another more recent low quality trial showing benefit for IM-lidocaine for chronic MND in the short-term, but this did not change the ICON review findings. In addition, we found new data for medial branch block with steroid at 2 year follow-up which was also statistically similar to the 1 year follow-up data included in the ICON review.

\section{CONCLUSIONS}

\section{Implications for Practice}

Limited conclusions can be drawn from this review. There was very low quality evidence favoring IM-lidocaine injection for chronic mechanical neck pain and nerve block anaesthetic for cervicogenic headache. There was moderate evidence of no difference between steroid and control injections for mechanical neck pain. The remaining eight trials of low or very low quality studying epidural corticosteroid, intracutaneous carbon dioxide or water injections, transforaminal corticosteroid and sphenopalatine ganglion block, showed no benefit of injection over control or placebo. The use of medicinal injections for MNDs cannot be supported based on the current evidence database.

\section{Implications for Research}

Future high quality research is required to make evidence based conclusions on the value of physician-delivered injections to treat MNDs. These trials should include larger 
sample sizes and be conducted over multiple centres to increase validity and generalizability.

Clinical applicability and relevance can be addressed by more effectively describing the patient population and interventions in detail, including the qualifications and training of those administering the intervention. It would be beneficial to include outcome measures on function/disability, quality of life, patient satisfaction and global perceived effect in addition to pain. Finally, reporting on adverse events will inform conclusions on benefit $v s$ harm of medicinal injections for MNDs.

\section{CONFLICT OF INTEREST}

The authors confirm that this article content has no conflict of interest.

\section{ACKNOWLEDGEMENTS}

This work was supported by Centric Health-LifeMark Industry Partner Grant. This is an interdisciplinary care service.

\section{APPENDIX A: MEDLINE SEARCH STRATEGY. Search Details: COG Injection (Neck Pain)-2012 \\ OVID-Medline}

1. Neck Pain/

2. exp Brachial Plexus Neuropathies/

3. exp neck injuries/ or exp whiplash injuries/

4. cervical pain.mp.

5. neckache.mp.

6. whiplash.mp.

7. cervicodynia.mp.

8. cervicalgia.mp.

9. brachialgia.mp.

10. brachial neuritis.mp.

11. brachial neuralgia.mp.

12. neck pain.mp.

13. neck injur*.mp.

14. brachial plexus neuropath*.mp.

15. brachial plexus neuritis.mp.

16. thoracic outlet syndrome/ or cervical rib syndrome/

17. Torticollis/

18. exp brachial plexus neuropathies/ or exp brachial plexus neuritis/

19. cervico brachial neuralgia.ti,ab.

20. cervicobrachial neuralgia.ti,ab.

21. (monoradicul* or monoradicl*).tw.

22. or/1-21

23. exp headache/ and cervic*.tw.

24. exp genital diseases, female/

25. genital disease*.mp.

26. or $/ 24-25$

27. 23 not 26

28. 22 or 27

29. neck/

30. neck muscles/

31. exp cervical plexus/

32. exp cervical vertebrae/

33. atlanto-axial joint/

34. atlanto-occipital joint/

35. Cervical Atlas/

36. spinal nerve roots/

37. exp brachial plexus/

38. (odontoid* or cervical or occip* or atlant*).tw.
39. axis/ or odontoid process/

40. Thoracic Vertebrae/

41. cervical vertebrae.mp.

42. cervical plexus.mp.

43. cervical spine.mp.

44. (neck adj3 muscles).mp.

45. (brachial adj3 plexus).mp.

46. (thoracic adj3 vertebrae).mp.

47. neck.mp.

48. (thoracic adj3 spine).mp.

49. (thoracic adj3 outlet).mp.

50. trapezius.mp.

51. cervical.mp.

52. cervico*.mp.

53.51 or 52

54. exp genital diseases, female/

55. genital disease*.mp.

56. $\exp *$ Uterus/

57.54 or 55 or 56

58.53 not 57

59.29 or 30 or 31 or 32 or 33 or 34 or 35 or 36 or 37 or 38 or 39 or 40 or 41 or 42 or 43 or 44 or 45 or 46 or 47 or 48 or 49 or 50 or 58

60. exp pain/

61. exp injuries/

62. pain.mp.

63. ache.mp.

64. sore.mp.

65. stiff.mp.

66. discomfort.mp.

67. injur*.mp.

68. neuropath*.mp.

69. or/60-68

70.59 and 69

71. Radiculopathy/

72. exp temporomandibular joint disorders/ or exp temporomandibular joint dysfunction syndrome/

73. myofascial pain syndromes/

74. exp "Sprains and Strains"/

75. exp Spinal Osteophytosis/

76. $\exp$ Neuritis/

77. Polyradiculopathy/

78. exp Arthritis/

79. Fibromyalgia/

80. spondylitis/ or discitis/

81. spondylosis/ or spondylolysis/ or spondylolisthesis/

82. radiculopathy.mp.

83. radiculitis.mp.

84. temporomandibular.mp.

85. myofascial pain syndrome*.mp.

86. thoracic outlet syndrome*.mp.

87. spinal osteophytosis.mp.

88. neuritis.mp.

89. spondylosis.mp.

90. spondylitis.mp.

91. spondylolisthesis.mp.

92. or/71-91

93.59 and 92

94. exp neck/

95. exp cervical vertebrae/

96. Thoracic Vertebrae/

97. neck.mp.

98. (thoracic adj3 vertebrae).mp.

99. cervical.mp.

100. cervico*.mp.

101.99 or 100 
102. exp genital diseases, female/

103. genital disease*.mp.

104. exp *Uterus/

105. or/102-104

106. 101 not 105

107. (thoracic adj3 spine).mp.

108. cervical spine.mp.

109. 94 or 95 or 96 or 97 or 98 or 106 or 107 or 108

110. Intervertebral Disk/

111. (disc or discs).mp.

112. (disk or disks).mp.

113. 110 or 111 or 112

114. 109 and 113

115. herniat*.mp

116. slipped.mp.

117. prolapse*.mp.

118. displace*.mp.

119. degenerat*.mp.

120. (bulge or bulged or bulging).mp.

121.115 or 116 or 117 or 118 or 119 or 120

122. 114 and 121

123. intervertebral disk degeneration/ or intervertebral disk displacement/

124. intervertebral disk displacement.mp.

125. intervertebral disc displacement.mp.

126. intervertebral disk degeneration.mp.

127. intervertebral disc degeneration.mp.

128. 123 or 124 or 125 or 126 or 127

129. 109 and 128

130.28 or 70 or 93 or 122 or 129

131. animals/ not (animals/ and humans/)

132. 130 not 131

133. exp *neoplasms/

134. exp *wounds, penetrating/

135. 133 or 134

136. 132 not 135

137. (needl* or acupuncture or inject*)

138. *aine/ tox.

139. block*

140. or/137-139

141.136 and 140

142. exp randomized controlled trials as topic/

143. randomized controlled trial.pt.

144. controlled clinical trial.pt.

145. (random* or sham or placebo*).tw.

146. placebos/

147. random allocation/

148. single blind method/

149. double blind method/

150. ((singl* or doubl* or trebl* or tripl*) adj25 (blind* or dumm* or mask*)).ti,ab

151. (rct or rets).tw.

152. (control* adj2 (study or studies or trial*)).tw.

153. or $/ 142-152$

154. guidelines as topic/

155. practice guidelines as topic/

156. guideline.pt.

157. practice guideline.pt.

158. (guideline? or guidance or recommendations).ti.

159. consensus.ti.

160. or/154-159

161. meta-analysis/

162. exp meta-analysis as topic/

163. (meta analy* or metaanaly* or met analy* or metanaly*).tw.

164. review literature as topic/

165. (collaborative research or collaborative review* or collaborative overview*).tw.

166. (integrative research or integrative review* or intergrative

overview*).tw.

167. (quantitative adj3 (research or review* or overview*)).tw.

168. (research integration or research overview*).tw.

169. (systematic* adj3 (review* or overview*)).tw.

170. (methodologic* adj3 (review* or overview*)).tw.

171. exp technology assessment biomedical/

172. (hta or thas or technology assessment*).tw.

173. ((hand adj2 search*) or (manual* adj search*)).tw.

174. ((electronic adj database*) or (bibliographic* adj database*)).tw.

175. ((data adj2 abstract*) or (data adj2 extract*)).tw.

176. (analys* adj3 (pool or pooled or pooling)).tw.

177. mantel haenszel.tw.

178. (cohrane or pubmed or pub med or medline or embase or psycinfo or

psyclit or psychinfo or psychlit or cinahl or science citation indes).ab.

179. or/161-178

180. 153 or 160 or 179

181. 141 and 180

\section{APPENDIX B}

\section{Excluded Studies}

\begin{tabular}{|c|c|}
\hline Study & Reason for Exclusion \\
\hline Barnsley 1994 [42] & Lack of proper control \\
\hline Bracker 2008 [43] & Intervention used was Botox \\
\hline Byrn 1993 [44] & Lack of proper control \\
\hline Carroll 2008 [45] & Intervention used was Botox \\
\hline Castagnera $1994[46]$ & Lack of proper control \\
\hline Childer 2005 [47] & Intervention used was oral medication \\
\hline Dreyfus 2006 [48] & Lack of proper control \\
\hline Evans 2003 [49] & Intervention used was oral medication \\
\hline Ga $2007[50]$ & Lack of proper control \\
\hline Kaya 2009 [51] & Lack of proper control \\
\hline Ketenci 2009 [52] & Lack of proper control \\
\hline Khwaja 2010 [53] & Intervention used was oral medication \\
\hline Lemming 2005 [54] & Intervention applied through intravenous \\
\hline Lemming 2007 [55] & Intervention applied through intravenous \\
\hline Lew 2008 [56] & Intervention used was Botox \\
\hline Ma 2008 [57] & Intervention used was oral medication \\
\hline McReynolds 2005 [58] & Lack of proper control \\
\hline Miller 2009 [59] & Intervention used was Botox \\
\hline Nikander 2006 [60] & Intervention used was exercise \\
\hline Pasqualucci 2007 [61] & Interventions given at different time periods \\
\hline Pato 2010 [62] & Lack of proper control \\
\hline Petterson 1998 [63] & Intervention used was infusion \\
\hline Stav 1993 [64] & Lack of proper control \\
\hline Thomas 1991 [65] & Intervention used was oral medication \\
\hline Tsai 2009 [66] & Lack of proper control \\
\hline Zhang 2005 [67] & Lack of proper control \\
\hline
\end{tabular}




\section{REFERENCES}

[1] Cote P, van der Velde G, Cassidy JD, et al. The burden and determinants of neck pain in workers: Results of The Bone and Joint Decade 2000-2010 Task Force on Neck Pain and its Associated Disorders. Spine 2008; 33(4 Suppl): S60-S74.

[2] Hogg-Johnson S, van der Velde G, Carroll LJ, et al. The burden and determinants of neck pain in the general population: Results of The Bone and Joint Decade 2000-2010 Task force on neck pain and its associated disorders. Spine 2008; 33(4 Suppl): S39-S51.

[3] Croft PR, Lewis M, Papageorgiou AC, et al. Risk factors for neck pain: a longitudinal study in the general population. Pain 2001;9(3):317-25.

[4] Makela M, Heliovaara M, Sievers K, Impivaara O, Knekt P, Aromaa A. Prevalence, determinants, and consequences of chronic neck pain in Finland. Am J Epidemiol 1991; 134(11): 1356-67.

[5] Picavet HS, Schouten JS. Musculoskeletal pain in the Netherlands: prevalence, consequences and risk groups, the $\mathrm{DMC}(3)$-study. Pain 2003; 102(1-2): 167-78

[6] Cote P, Cassidy JD, Carroll LJ, Kristman V. The annual incidence and course of neck pain in the general population: A populationbased cohort study. Pain 2004; 112(3): 267-73.

[7] Cassidy JD, Carroll LJ, Cote P, Lemstra M, Berglund A, Nygren A. Effect of eliminating compensation for pain and suffering on the outcome of insurance claims for whiplash injury. N Engl J Med 2000; 342(16): 1179-86.

[8] Radanov BP, Sturzenegger M, De SG, Schnidrig A. Relationship between early somatic, radiological, cognitive and psychosocial findings and outcome during a one-year follow-up in 117 patients suffering from common whiplash. Br J Rheumatol 1994; 33(5): 442-8.

[9] Holm LW, Carroll LJ, Cassidy JD, Skillgate E, Ahlbom A. Widespread pain following whiplash-associated disorders: incidence, course, and risk factors. J Rheumatol 2007; 34(1): 193200.

[10] Borghouts JA, Koes BW, Vondeling H, Bouter LM. Cost-of-illness of neck pain in The Netherlands in 1996. Pain 1999;80(3):629-36.

[11] Carragee EJ, Hurwitz EL, Cheng I, et al. Treatment of neck pain: Injections and surgical interventions: Results of the Bone and Joint Decade 2000-2010 Task Force on neck pain and its associated disorders. Spine 2008; 33(4 Suppl): S153-69.

[12] Drewes AM, Jensen RD, Nielsen LM, et al. Differences between opioids: Pharmacological, experimental, clinical and economical perspectives. Br J Clin Pharmacol 2013; 75(1): 60-78.

[13] Smith C. Pharmacology of local anaesthetic agents. Br J Hosp Med 1994; 52(9): 455-60.

[14] Peloso PM, Gross AR, Haines TA, Trinh K, Goldsmith CH, Aker $P$. Medicinal and injection therapies for mechanical neck disorders: a Cochrane systematic review. J Rheumatol 2006; 33: 957-67.

[15] Langevin P, Peloso PM, Lowcock J, et al. Botulinum toxin for subacute/chronic neck pain. Cochrane Database Syst Rev 2011; (7): CD008626.

[16] Peloso P, Khan M, Gross AR, et al. Pharmacological interventions including medical injections for neck pain: An overview as part of the ICON Project. Open Orthop J, [in press].

[17] Beattie P. Measurement of health outcomes in the clinical setting: Applications to physiotherapy. Physiother Theory Pract 2001; 17: 173-85.

[18] Finch E, Brooks D, Stratford P, Mayo N. Physical rehabilitation outcome measures: A guide to enhanced clinical decision making. $2^{\text {nd }}$ ed. Philadelphia: Lippincott Williams and Wilkins, 2002.

[19] Cicchetti DV. Assessing inter-rater reliability for rating scales: resolving some basic issues. Br J Psychiatry 1976;129:452-6.

[20] Furlan AD, Pennick V, Bombardier C, van Tulder M, Editorial Board Cochrane Back Review Group. 2009 Updated method guidelines for systematic reviews in the Cochrane Back Review Group. Spine 2009; 34(18): 1929-41.

[21] Graham N, Haines $\mathrm{T}$, Goldsmith $\mathrm{CH}$, et al. Reliability of 3 assessment tools used to evaluate randomized controlled trials for treatment of neck pain. Spine 2012; 37(6): 515-22.

[22] Higgins JPT, Green S, Eds. Cochrane Handbook for Systematic Reviews of Interventions Version 5.0.2 [updated September 2009]. The Cochrane Collaboration, 2009. Available from: www.cochrane-handbook.org

[23] Farrar JT, Young JP Jr, LaMoureaux L, Worth JL, Poole RM. Clinical importance of changes in chronic pain intensity measured on an 11-point numerical rating scale. Pain 2001; 94: 149-58.
[24] Felson DT, Anderson JJ, Boers M, et al. American College of Rheumatology: Preliminary definition of improvement in rheumatoid arthritis. Arthritis Rheum 1995; 38(6): 727-35.

[25] Cleland JA, Childs JD, Whitman JM. Psychometric properties of the neck disability index and the numeric pain rating scale in patients with mechanical neck pain. Arch Phys Med Rehabil 2008; 89: 69-74.

[26] MacDermid JC, Walton DM, Avery S, et al. Measurement properties of the neck disability index: a systematic review. J Orthop Sports Phys Ther 2009; 39(5): 400-17.

[27] Kendal MG, Stuart A. The advanced theory of statistics: Distribution theory. $2^{\text {nd }}$ ed. vol. 1 . New York: Hafner Publishing Co, 1963.

[28] Manchikanti L, Singh V, Falco FJ, Cash KA, Fellows B. Comparative outcomes of a 2-year follow-up of cervical medial branch blocks in management of chronic neck pain: a randomized, double-blind controlled trial. Pain Physician 2010a; 13(5): 437-50.

[29] Manchikanti L, Singh V, Falco FJ, Cash KM, Fellows B. Cervical medial branch blocks for chronic cervical facet joint pain: a randomized, double-blind, controlled trial with one-year follow-up. Spine 2008; 33(17): 1813-20.

[30] Manchikanti L, Damron K, Cash K, Manchukonda R, Pampati V. Therapeutic cervical medial branch blocks in managing chronic neck pain: a preliminary report of a randomized, double-blind, controlled trial: clinical trial NCT0033272. Pain Physician 2006; 9(4): 333-46.

[31] Ay S, Evcik D, Tur BS. Comparison of injection methods in myofascial pain syndrome: a randomized controlled trial. Clin Rheumatol 2010; 29(1): 19-23.

[32] Kamanli A, Kaya A, Ardicoglu O, Ozgocmen S, Ozkurt Zenigin F, Bayik Y. Comparison of lidocaine injection, botulinum toxin injection, and dry needling to trigger points in myofascial pain syndrome. Rheumatol Int 2005; 25: 604-11.

[33] Esenyel M, Caglar N, Aldemir T. Treatment of myofascial pain. Am J Phys Med Rehabil 2000; 79(1): 48-52.

[34] Hong CZ. Lidocaine injection $v s$ dry needling to myofascial trigger point: The importance of the local twitch response. Am J Phys Med Rehabil 1994; 73(4): 256-63.

[35] Naja ZM, El-Rajab M, Al-Tannir MA, Ziade FM, Tawfik OM. Occipital nerve blockade for cervicogenic headache: a double-blind randomized controlled clinical trial. Pain Pract 2006; 6(2): 89-95.

[36] Terzi T, Karakurum B, Ucler S, Inan LE, Tulumay C. Greater occipital nerve blockade in migraine, tension-type headache and cervicogenic headache. J Headache Pain 2002; 3: 137-41.

[37] Ferrante FM, Kaufman AG, Dunbar SA, Cain CF, Cherukuri S Sphenopalatine ganglion block for the treatment of myofascial pain of the head, neck and shoulders. Reg Anesth Pain Med 1998; 23(1): 30-6.

[38] Manchikanti L, Cash KA, Pampati V, Wargo BW, Malla Y. Cervical epidural injections in chronic discogenic neck pain without disc herniation or radiculitis: preliminary results of a randomized, double-blind, controlled trial. Pain Physician 2010b; 13(4): E265-78.

[39] Brockow T, Heissner T, Franke A, Resch K. Evaluation of the efficacy of subcutaneous carbon dioxide insufflations for treating acute non-specific neck pain in general practice: A sham controlled randomized trial. Eur J Pain 2008; 12(1): 9-16.

[40] Sand T, Bovim G, Held G. Intracutaneous sterile water injections do not relieve pain in cervicogenic headache. Acta Neurol Scand 1992; 86: 526-8.

[41] Anderberg L, Annertz M, Persson L, Brandt L, Säveland H. Transforaminal steroid injection for the treatment of cervical radiculopathy: a prospective and randomised study. Eur Spine J 2007; 16(3): 321-8.

[42] Barnsley L, Lord SM, Wallis BJ, Bogduk N. Lack of effect of intraarticular corticosteroids for chronic pain in the cervical zygapophyseal joints. N Engl J Med 1994; 330(15): 1047-50.

[43] Braker C, Yariv S, Adler R, Badarny S, Eisenberg E. The analgesic effect of botulinum-toxin A on postwhiplash neck pain. Clin J Pain 2008; 24: 5-10.

[44] Byrn C, Olsson I, Falkheden L, et al. Subcutaneous sterile water injections for chronic neck and shoulder pain following whiplash injuries. Lancet 1993; 341(8843): 449-52.

[45] Carroll A, Barnes M, Comisky C. A prospective randomized controlled study of the role of botulinum toxin in whiplashassociated disorder. Clin Rehabil 2008; 22: 513-9. 
[46] Castagnera L, Maurette P, Pointellart V, Vital JM, Erny P, Stenegas J. Long-term results of cervical epidural steroid injection with and without morphine in chronic cervical radicular pain. Pain 1994; 58: 239-43.

[47] Childers MK, Borenstein D, Brown RL, et al. Low-dose cyclobenzaprine $v s$ combination therapy with ibuprofen for acute neck or back pain with muscle spasm: a randomized trial. Curr Med Res Opin 2005; 21(9): 1485-93.

[48] Dreyfuss P, Baker R, Bogduk N. Comparative effectiveness of cervical transforaminal injections with particulate and nonparticulate corticosteroid preparations for cervical radicular pain. Pain Med 2006; 7(3): 237-42.

[49] Evans R, Bronfort G, Bittel S, Anderson AV. A pilot study for a randomized clinical trial assessing chiropractic care, medical care, and self-care education for acute and subacute neck pain patients. J Manipulative Physiol Ther 2003; 26: 403-11.

[50] Ga H, Choi JH, Park CH, Yoon HJ. Acupuncture needling vs lidocaine injection of trigger points in myofascial pain syndrome in elderly patients--a randomised trial. Acupunct Med 2007; 25(4): 130-6.

[51] Kaya A, Kamanli A, Ardicoglu O, Ozgocmen S, Ozkurt-Zengin F, Bayik Y. Direct current therapy with/without lidocaine iontophoresis in myofascial pain syndrome. Bratisl Lek Listy 2009; 110(3): 185-91.

[52] Ketenci A, Basat H, Esmaeilzadeh S. The efficacy of topical thiocolchicoside (Muscoril) in the treatment of acute cervical myofascial pain syndrome: a single-blind, randomized, prospective, phase IV clinical study. Agri 2009; 21(3): 95-103.

[53] Khwaja SM, Minnerop M, Singer AJ. Comparison of ibuprofen, cyclobenzaprine or both in patients with acute cervical strain: a randomized controlled trial. CJEM 2010; 12(1): 39-44.

[54] Lemming D, Sörensen J, Graven-Nielsen T, Arendt-Nielsen L, Gerdle B. The responses to pharmacological challenges and experimental pain in patients with chronic whiplash-associated pain. Clin J Pain 2005; 21(5): 412-21.

[55] Lemming D, Sörensen J, Graven-Nielsen T, Lauber R, ArendtNielsen L, Gerdle B. Managing chronic whiplash associated pain with a combination of low-dose opioid (remifentanil) and NMDAantagonist (ketamine). Eur J Pain 2007; 11(7): 719-32.
[56] Lew HL, Lee EH, Castaneda A, Klima R, Date E. Therapeutic Use of Botulinum Toxin A in Treating Neck and Upper-Back Pain of Myofascial Origin: A pilot study. Arch Phys Med Rehabil 2008; 89: 75-80.

[57] Ma K, Jiang W, Zhou Q, Du DP. The efficacy of oxycodone for management of acute pain episodes in chronic neck pain patients Int J Clin Pract 2008; 62(2): 241-7.

[58] McReynolds TM, Sheridan BJ. Intramuscular Ketorlac vs osteopathic manipulative treatment in the management of acute neck pain in the emergency department: A randomized clinical trial. J Am Osteopath Assoc 2005; 105(2): 57-68.

[59] Miller D, Richardson D, Eisa M, Bajwa RJ, Jabbari B. Botulinum neurotoxin-A for treatment of refractory neck pain: a randomized, double-blind study. Pain Med 2009; 10(6): 1012-7.

[60] Nikander R, Mälkiä E, Parkkari J, Heinonen A, Starck H, Ylinen J. Dose-response relationship of specific training to reduce chronic neck pain and disability. Med Sci Sports Exerc 2006; 38(12): 206874.

[61] Pasqualucci A, Varrassi G, Braschi A, et al. Epidural local anesthetic plus corticosteroid for the treatment of cervical brachial radicular pain: single injection $v s$ continuous infusion. Clin $\mathrm{J}$ Pain 2007; 23(7): 551-7.

[62] Pato U, Di Stefano G, Fravi N, et al. Comparison of randomized treatments for late whiplash. Neurology 2010; 74(15): 1223-30.

[63] Pettersson K, Toolanen G. High-dose methylprednisolone prevents extensive sick leave after whiplash injury, a prospective, randomized, double-blind study. Spine 1998; 23(9): 984-98.

[64] Stav A, Ovadia L, Sternberg A, Kaadan M, Weksler N. Cervical epidural steroid injection for cervicobrachialgia. Acta Anaesthesiol Scand 1993; 37: 562-6.

[65] Thomas M, Eriksson SV, Lundeberg T. A comparative study of Diazepam and acupuncture in patients with osteoarthritis pain: A placebo controlled study. Am J Chin Med 1991; 19(2): 95-100.

[66] Tsai CT, Hsieh LF, Kuan TS, Kao MJ, Hong CZ. Injection in the cervical facet joint for shoulder pain with myofascial trigger points in the upper trapezius muscle. Orthopedics 2009; 32(8): pii: orthosupersite.com/view.asp?rID=41914.

[67] Zhang W, Zheng J, Zhao X. Treatment of arterial-type cervical spondylosis with acupoint-injection combined with massage manipulation. Int J Clin Acupunct 2005; 14(4): 261-5.

(c) Gross et al.; Licensee Bentham Open

This is an open access article licensed under the terms of the Creative Commons Attribution Non-Commercial License (http://creativecommons.org/licenses/by-nc/3.0/) which permits unrestricted, non-commercial use, distribution and reproduction in any medium, provided the work is properly cited. 